Ann. Génét. Sél. anim., I977, 9 (3), 353-377.

\title{
Dix ans d'une expérience de sélection individuelle sur des verrats utilisés en insémination artificielle
}

\section{I. -.- Réponses observées sur des caractères de croissance de carcasse et de qualité de viande}

\author{
L. OLLIVIER
}

Avec la collaboration technique de Marie-Reine Langlors, J. Gruand, D. Tastu et C. Felgines.

Station de Génétique quantitative et appliquée, Centre national de Recherches zootechniques, I.N.R.A., $7835^{\circ}$ Jouy en Josas

\section{Résumé}

Cet article décrit le dispositif et donne les résultats d'une expérience de sélection réalisée dans le cadre d'un centre d'insémination artificielle porcine I.N.R.A. situé à Rouillé (Vienne). A partir de ro verrats "fondateurs " $\left(\mathrm{G}_{0}\right)$ mis en service en novembre 1965,9 générations successives de verrats $\left(G_{1}\right.$ à $\left.G_{9}\right)$ ont été sélectionnées, soient 84 verrats au total, et $273^{2}$ descendants (mâles castrés et femelles) de ces 94 verrats ont été contrôlés entre 1966 et 1975. Les jeunes verrats mis à l'insémination chaque année (au mois de novembre) étaient les fils de ceux mis en service un an plus tôt et de truies choisies au hasard dans la zone d'insémination. Ainsi aucun verrat extérieur à cette zone n'a été introduit au cours de l'expérience. La sélection des verrats était basée sur un indice individuel combinant vitesse de croissance de 30 à $80 \mathrm{~kg}$ et gras dorsal à $80 \mathrm{~kg}$, la consommation alimentaire individuelle n'ayant été prise en considération que jusqu'à 1968. Parallèlement à l'épreuve individuelle des verrats, la descendance des mâles sélectionnés l'année précédente et celle des mâles restants parmi ceux sélectionnés deux ans plus tôt étaient soumises simultanément à un engraissement de 30 à $\mathrm{too} \mathrm{kg}$, avec alimentation à volonté au nourrisseur, de manière à mesurer, pour la vitesse de croissance l'efficacité alimentaire la composition corporelle et la qualité de la viande, les réponses à la sélection pratiquée.

L'analyse des résultats, par la méthode des moindres carrés, met en évidence, pour la plupart des variables, des effets non-génétiques hautement significatifs, tels que le sexe, le bâtiment d'engraissement (pour les caractères de croissance et de carcasse) ou la date d'abattage (pour la qualité de la viande). Les différences génétiques entre les générations paternelles sont dans l'ensemble plus importantes et plus significatives qu'entre les cohortes maternelles. Comme les périodes étudiées sont I960-I973 et I966-1974 respectivement pour les mères et pour les pères, cette différence traduit en fait une accélération du progrès génétique depuis 1966, attribuable sans doute à la sélection pratiquée sur les verrats. Les gains génétiques annuels sur les pères ont été estimés à environ $\mathrm{I}_{4} \mathrm{~g}$ par jour en vitesse de croissance, $-0,04$ point d'indice de consommation et $-0,5 \mathrm{~mm}$ d'épaisseur de lard sur la carcasse. Ces gains correspondent à une économie annuelle de $0,2 \mathrm{~kg}$ d'aliment par $\mathrm{kg}$ de tissu maigre produit et à une augmentation annuelle de la vitesse de croissance du tissu maigre de $7 \mathrm{~g}$ par jour. Quant à la qualité de ce tissu maigre, appréciée par le $\mathrm{pH}$ ultime sur 4 muscles, la couleur et le pouvoir de rétention d'eau sur 2 muscles, 
rien n'indique qu'elle se soit dégradée au cours de l'expérience, si l'on excepte toutefois la couleur du muscle long vaste dont l'évolution, du côté maternel, est défavorable. Particulièrement remarquable est l'augmentation régulière et hautement significative du poids de la tête depuis le début de l'expérience; cela traduit une maturité physiologique moins avancée au poids d'abattage de $100 \mathrm{~kg}$ de l'animal maigre à croissance rapide que tend à produire le type de sélection pratiqué dans cette expérience. On doit s'attendre, en conséquence, à une diminution du poids de la carcasse, diminution qui n'a cependant pas été observée dans cette expérience. La dernière génération considérée dans cette étude $\left(\mathrm{G}_{9}\right)$ a été comparée au Large White national contemporain par Sellier (Journées de la Recherche Porcine en France, I977, p. 85): si en efficacité de production du tissu maigre la $\mathrm{G}_{9}$ est voisine génétiquement du Large White, la $\mathrm{G}_{9}$ est nettement supérieure en vitesse de croissance du tissu maigre et aussi en qualité de ce tissu.

\section{Introduction}

Les méthodes de sélection du Porc ont subi, au cours de ces 20 dernières années dans la pluplart des pays occidentaux, une profonde transformation qui a consisté dans le passage de l'épreuve de descendance, préconisée par les Danois depuis le début de ce siècle, à l'épreuve individuelle, rendue possible par les techniques permettant l'estimation objective de la composition corporelle du porc vivant (Hazel et Kline, I952; Dumont, I957). En France, ce passage s'est effectué progressivement au cours de la décade I966-1975. Une idée de l'ampleur de cette évolution dans notre pays est donnée par la comparaison du nombre de verrats contrôlés sur descendance en I960, qui était de I8, et du nombre de verrats contrôlés individuellement en 1975 qui dépassait 6 ooo, alors que le nombre de verrats contrôlés sur descendance la même année n'était que de 75 .

D'autre part, les paramètres génétiques des populations porcines européennes (Smith et al., I962; Jonsson, I965; Ollivier, I970a; Pfleiderer, I973 et I974; HANSET et VAN SNICK, I972 et 1973) montrent qu'il est possible d'améliorer simultanément la vitesse de croissance, l'efficacité alimentaire et la composition corporelle, car les corrélations génétiques entre ces caractères sont, dansl'ensemble, soit favorables soit faibles. Une sélection simultanée sur vitesse de croissance et adiposité doit donc être efficace. De nombreuses expériences de sélection sur le Porc ont montré l'efficacité de la sélection individuelle sur l'un ou l'autre de ces deux caractères (Fredeen, I958; RAhNefeld, I97I; Hetzer et Miller, I973). Par contre, la sélection simultanée sur plusieurs caractères, inclus dans un indice, a jusqu'à présent été peu expérimentée dans l'espèce porcine. Citons cependant des résultats récents norvégiens (VANGEn, I974; BAKKE et STANDAL, I975) et de l'ABRO à Edimbourg (WeBb et KING, 1976).

L'expérience qui va être décrite a commencé en I965. Elle avait pour but d'étudier expérimentalement l'efficacité d'une sélection individuelle des verrats sur un indice incluant un nombre de limité caractères simples (2 ou 3). L'objet de ce mémoire, qui reprend des résultats préliminaires antérieurement publiés (OlLIVIER, I970 $b$ et I97I), est d'étudier l'évolution qui s'est produite au cours des dix premières années de l'expérience - ce qui correspond à 9 générations successives de sélection sur les verrats - pour des caractères de croissance de carcasse et de qualité de viande. Ce premier article donne le détail du schéma expérimental appliqué, des méthodes d'estimation utilisées et des résultats obtenus. Un deuxième article sera consacré à l'interprétation génétique de ces résultats. 


\section{Matériel et méthodes}

\section{I. - Schéma expérimental}

a) Sélection des verrats.

Un Centre expérimental d'insémination artificielle porcine a été ouvert par l'INRA en I96o à Rouillé (Vienne). La population porcine concernée comptait alors environ 5 ooo truies de type Large White, réparties dans de nombreux petits élevages des deux départements de la Vienne et des Deux-Sèvres. Jusqu'en I965, des verrats Large White en provenance d'élevages du Herd-Book de cette race et, pour la plupart, fils de verrats contrôlés sur descendance ont été utilisés. En novembre 1965 , dix verrats de ce type, dont deux en provenance d'un premier essai de contrôle individuel effectué à Jouy-en-Josas, ont été mis à l'insémination et ont constitué la génération des fondateurs $\left(G_{0}\right)$. A partir de cette date, les verrats mis à l'insémination en novembre chaque année étaient les fils de ceux mis en service en novembre de l'année précédente et de truies choisies au hasard dans la zone d'insémination. Ainsi, aucun verrat extérieur à cette zone n'a été introduit au cours de l'expérience.

A partir de I966, chaque année un lot de jeunes mâles nés en mars a été soumis à une épreuve individuelle ccmprenant des contrôles de croissance (et de consommation individuelle d'aliment de I966 à I 668 ) entre 30 et $80 \mathrm{~kg}$, et des mesures d'épaisseur de lard vers $80 \mathrm{~kg}$. Les 3 mesures de gain moyen, quotidien d'indice de consommation et d'épaisseur de lard dorsal étaient combinées en un indice de sélection $I_{1}$, puis à partir de $I_{969}$ en un indice simplifié $I_{2}$ excluant l'indice de consommation; les modalités d'établissement de ces indices sont présentées au tableau I. Le nombre de verrats contrôlés chaque année, qui était voisin de 50 les trois premières années, a été ensuite augmenté, grâce au remplacement du contrôle individuel de la consommation, au cours de deux repas journaliers, par une alimentation à volonté au nourrisseur par groupe de ro. Comme le nombre de verrats sélectionnés chaque année était de Io (à l'exception de trois années où ce chiffre a été ramené à 8 ) l'intensité de sélection s'est régulièrement accrue au cours de l'expérience. D'autre part la sélection, qui se faisait intra-famille de père de I966 à I97I, est devenue purement massale depuis I972. Au total de I966 à I975, 850 verrats ont été soumis à l'épreuve individuelle et 84 ont été retenus pour constituer les générations $G_{1}$ à $G_{9}$.

\section{b) Mesure des réponses à la sélection.}

Parallèlement à l'épreuve individuelle des verrats, des mâles castrés et des femelles étaient mis à l'engraissement à $30 \mathrm{~kg}$ et abattus à un poids vif voisin de Ioo kg. Chaque année, cet engraissement permettait de comparer la descendance des verrats sélectionnés l'année précédente à celle des verrats restants parmi ceux sélectionnés deux ans plus tôt. Ainsi, en I 967 , la descendance des verrats fondateurs $G_{0}$ a-t-elle été comparée à celle de leurs fils $G_{1}$ sélectionnés en I 966 , et ainsi de suite jusqu'à $I 975$ où la comparaison portait sur $G_{8}$ et $G_{9}$; ce shéma expérimental, qui est une application de la méthode des accouplements répétés (repeat-mating scheme) de GoODWIN et coll. (I955), a permis de mesurer les réponses à la sélection 


\section{TABLEAU I}

Les indices de sélection paramètres utilisés

(Parameters used to establish the selection indices)

\begin{tabular}{|c|c|c|c|c|c|}
\hline \multirow{2}{*}{ Variables (I) } & \multirow{2}{*}{$\begin{array}{l}\text { Valeur économique } \\
\text { relative par unité } \\
\text { d'écart-type } \\
\text { (Relative economic } \\
\text { value per standard- } \\
\text { deviation unit) }\end{array}$} & \multicolumn{3}{|c|}{$\begin{array}{c}\text { Paramètres génétiques }\left({ }^{*}\right) \\
\text { (Genetic parameters) }\end{array}$} & \multirow{2}{*}{$\begin{array}{l}\text { Écart-type } \\
\text { (Standard- } \\
\text { deviation) }\end{array}$} \\
\hline & & $\mathrm{X}_{1}$ & $\mathrm{X}_{2}$ & $\mathrm{X}_{3}$ & \\
\hline $\begin{array}{l}\text { Gain moyen quotidien }\left(\mathrm{X}_{1}\right) \\
\text { (Average daily gain) } . .\end{array}$ & I & 0,36 & $-0,7 \mathrm{I}$ & 0,13 & IOO \\
\hline $\begin{array}{l}\text { Indice de consommation } \\
\left.\left(\mathrm{X}_{2}\right) \text { (feed conversion }\right) .\end{array}$ & 2 & $-o, 64$ & 0,42 & 0,10 & 0,3 \\
\hline $\begin{array}{l}\text { Épaisseur de lard }\left(\mathrm{X}_{3}\right) \\
\text { (Backfat thickness).... }\end{array}$ & 2 & $0, \mathbf{I} \mathbf{r}$ & 0,07 & 0,57 & 3 \\
\hline
\end{tabular}

(*) Héritabilités dans la diagonale, corrélations phénotypiques et génétiques respectivement audessus et au-dessous. (Heritabilities in the diagonal, phenotypic and genetic correlations respectively above and below.)

\section{Caractéristiques des indices obtenus}

(Indices obtained)

\begin{tabular}{|c|c|c|c|}
\hline & & $\begin{array}{|lll|} & I_{1} & \\
\text { (utilisé de } & \text { I966 à I968) }\end{array}$ & 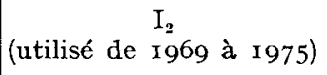 \\
\hline 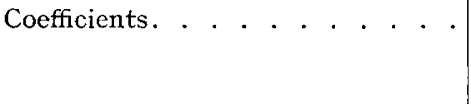 & $\begin{array}{l}X_{1} \\
X_{2} \\
X_{3}\end{array}$ & $\begin{array}{ll} & 0,01 \\
- & 6 \\
- & 0,8\end{array}$ & $\underset{0,5}{-O, O I}$ \\
\hline $\begin{array}{l}\text { Corrélation entre l'indice et la } \\
\text { valeur génétique globale (Correla- } \\
\text { tion between index and aggregate } \\
\text { breeding value) } . . \\
.\end{array}$ & & 0,68 & 0,64 \\
\hline $\begin{array}{l}\text { Réponse attendue par unité d'in- } \\
\text { tensité de sélection (Expected } \\
\text { response per unit selection intensity }\end{array}$ & $\begin{array}{l}\mathrm{X}_{1} \\
\mathrm{X}_{2} \\
\mathrm{X}_{3}\end{array}$ & $\begin{array}{ll} & \mathrm{I} 7 \\
- & 0,09 \\
- & \mathrm{I}, 2\end{array}$ & $\begin{array}{ll} & \mathrm{r} 8 \\
- & 0,06 \\
- & 1,4\end{array}$ \\
\hline
\end{tabular}

(i) $\mathrm{X}_{1}$ en $g ; \mathrm{X}_{2}$ en $\mathrm{kg}$ aliment $/ \mathrm{kg}$ de gain; $\mathrm{X}_{3}$ en $\mathrm{mm}\left(\mathrm{X}_{1}\right.$ in $\mathrm{g} ; \mathrm{X}_{2}$ in $\mathrm{kg}$ feed $/ \mathrm{kg}$ gain; $\mathrm{X}_{3}$ in $\left.\mathrm{mm}\right)$. Ces variables sont décrites dans le paragraphe "variables considérées ": la variable $\mathbf{X}_{3}$ a été corrigée pour les variations de poids vif autour de $80 \mathrm{~kg}$ en appliquant une correction de $0,2 \mathrm{~mm}$ par kg. (the variables are more completely described in text. $\mathrm{X}_{3}$ is corrected for liveweight variations using a correction of $0,2 \mathrm{~m}$ per $\mathrm{kg}$ ). 
pratiquée pour les caractères de croissance, d'efficacité alimentaire, de carcasse et, à partir de I969, pour la qualité de la viande.

A l'engraissement, les animaux étaient regroupés par loge d'environ ro individus de même génération, les loges attribuées aux deux générations mises en comparaison chaque année étant alternées à l'intérieur de chaque bâtiment. L'aliment était distribué à volonté au nourrisseur. La composition des aliments, qui étaient les mêmes pour les verrats, les mâles castrés et les femelles, est donnée au tableau 2.

Au total de rg66 à 1975,2732 porcs issus de 94 pères ont été soumis à ces contrôles.

TABLEAU 2

Composition des aliments

(Feed composition)

\begin{tabular}{|c|c|c|c|c|}
\hline & \multicolumn{2}{|c|}{ I 966-I967 } & \multicolumn{2}{|c|}{ I 968-I975 } \\
\hline & $\begin{array}{l}\text { Croissance } \\
\text { (Growth) }\end{array}$ & $\begin{array}{l}\text { Finition } \\
\text { (Finition) }\end{array}$ & $\begin{array}{c}\text { Croissance } \\
\text { (Growth) }\end{array}$ & $\begin{array}{l}\text { Finition } \\
\text { (Finition) }\end{array}$ \\
\hline 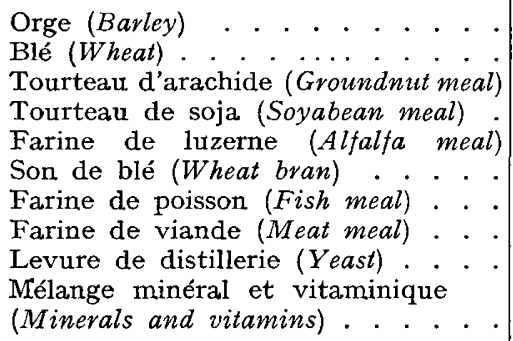 & $\begin{array}{r}38 \\
38 \\
4 \\
4 \\
5 \\
2 \\
2 \\
4 \\
3\end{array}$ & $\begin{array}{r}41 \\
42 \\
3 \\
3 \\
5 \\
\\
2 \\
2 \\
2\end{array}$ & $\begin{array}{r}73 \\
\mathbf{1 2} \\
3 \\
5 \\
4\end{array}$ & $\begin{array}{r}10 \\
5 \\
10\end{array}$ \\
\hline
\end{tabular}

Le passage de l'aliment croissance à l'aliment finition se faisait vers le poids de $60 \mathrm{~kg}$. (The change of diet was made around $60 \mathrm{~kg}$ liveweiht).

\section{2. - Variables considérées}

a) Sur les verrats candidats à la sélection.

$\mathrm{X}_{1}$ : Gain moyen quotidien de 30 à $80 \mathrm{~kg}$.

$\mathrm{X}_{2}$ : Indice de consommation individuel ( $\mathrm{kg}$ aliment $/ \mathrm{kg}$ de gain).

$\mathrm{X}_{3}$ : Moyenne de 6 épaisseurs de gras mesurées vers le poids de $80 \mathrm{~kg}$.

$\mathrm{X}_{4}$ : Moyenne de 6 épaisseurs de gras mesurées vers le poids de $40 \mathrm{~kg}$.

$\mathrm{X}_{5}$ : Moyenne de 6 épaisseurs de gras mesurées vers le poids de $60 \mathrm{~kg}$.

Les mesures $\mathrm{X}_{3}, \mathrm{X}_{4}$ et $\mathrm{X}_{5}$ ont été effectuées à partir de $\mathrm{r}_{96} 6$ à l'aide d'un appareil à ultra-sons KRAUTKRAMER. La mesure $\mathrm{X}_{3}$ a, de plus, été faite chaque année à partir de Ig66 à l'aide de la réglette métallique de HAZEL et Kline (I952). Cela 
a permis, à partir de I967, de vérifier l'accord entre les deux techniques pour $\mathrm{X}_{\mathbf{3}}$. Les épaisseurs de gras étaient mesurées en 6 points, situés symétriquement de part et d'autre de la ligne médiane, soit 2 à l'épaule, 2 au dos et 2 au rein (Ollivier, I965).

\section{b) Sur les descendants abattus.}

Ces animaux au terme de l'engraissement étaient abattus, à un poids vif voisin de Ioo kg, aux abattoirs Archaimbault à Celle-sur-Belle (Deux-Sèvres) et soumis le lendemain de l'abattage à la découpe parisienne normalisée et aux mesures de carcasse décrites par OllivieR ( I970 a), ainsi qu'à des mesures de qualité technologique de la viande (acidité, couleur et pouvoir de rétention d'eau). Les variables étudiées peuvent se classer en quatre catégories :

- la vitesse de croissance (GMQ) pendant l'engraissement de $30 \mathrm{~kg}$ environ jusqu'au poids d'abattage voisin de Ioo $\mathrm{kg}$ (variable $\mathrm{Y}_{\mathbf{1}}$ );

- les poids de la carcasse sans tête (poids net), de la tête et des différents morceaux : jambon, longe, poitrine, hachage, pieds, bardière, panne (variables $\mathrm{Y}_{\mathbf{2}}$ à $\mathrm{Y}_{10}$ );

- les mesures linéaires de longueur (atlas-pubis), et d'épaisseurs de lard au rein au dos et au cou (variables $\mathrm{Y}_{11}$ à $\mathrm{Y}_{14}$ ).

Les variables $\mathrm{Y}_{\mathbf{2}}$ à $\mathrm{Y}_{\mathbf{1 4}}$ ont été corrigées chaque année pour les variations de poids vif d'abattage en fonction des régressions linéaires estimées dans l'année correspondante.

- les mesures de qualité de la viande incluant :

$\times 4$ mesures de $p H$ (variables $\mathrm{Y}_{15}$ à $\mathrm{Y}_{18}$ ) sur les muscles adducteur (adductor femoris, AF), long vaste (biceps femoris, BF), fessier superficiel (gluteus superficialis, GS) et long dorsal (longissimus dorsi, LD), effectuées à l'aide d'un $\mathrm{pH}$ mètre portatif, EIL 30C;

$\times 2$ mesures de réflectance (variables $\mathrm{Y}_{19}$ à $\mathrm{Y}_{20}$ ) sur les muscles $\mathrm{BF}$ et $\mathrm{GS}$, effectuées à l'aide du réflectomètre Manuflex VERGE-Nicou;

$\times 2$ temps d'imbibition (variables $\mathrm{Y}_{21}$ et $\mathrm{Y}_{22}$ ) sur les muscles $\mathrm{BF}$ et GS, qui sont les temps nécessaires à un papier $\mathrm{pH}$, appliqué sur le muscle fraîchement coupé, pour changer de couleur entièrement, le temps d'observation étant limité à 3 minutes.

En plus de ces mesures individuelles, la consommation totale d'aliment par loge, le nombre de jours de présence et le gain de chaque porc de la loge (y compris les porcs éliminés en cours de contrôle) étaient enregistrés sur une période allant du jour où le poids moyen des porcs de la loge avoisinait $30 \mathrm{~kg}$ jusqu'au jour où le poids moyen (des porcs non éliminés) avoisinait $80 \mathrm{~kg}$. Trois variables de loge ont été considérées :

$\mathrm{L}_{1}$ : croissance moyenne journalière par porc qui est le rapport du gain de poids total à la durée totale de présence ;

$\mathrm{L}_{2}$ : indice de consommation moyen par porc, qui est le rapport de la consommation totale au gain de poids total;

$\mathrm{L}_{3}$ : consommation moyenne journalière par porc qui est le rapport de la consommation totale sur le nombre total de jours de présence des porcs de la loge. 


\section{3. - Méthode d'analyse}

a) Variables "verrats" $\left(\mathrm{X}_{1}, \ldots \mathrm{X}_{5}\right)$.

Un modèle linéaire additif, avec effets principaux et covariables, a été appliqué à ces variables. Les effets principaux considérés sont :

- l'année de contrôle, allant de I966 à I975 (ro niveaux);

- l'année de naissance de la mère, appelée cohorte maternelle, qui va de I959 à I973 (I5 niveaux). Une légère correction a été apportée à ce facteur pourtenir compte du fait que les animaux contrôlés chaque année $n$ sont nés en mars : leurs mères nées dans l'année $n$-I sont nécessairement nées dans les premiers mois de l'année, alors que les naissances des mères des années antérieures sont étalées uniformément sur les I2 mois de l'année. Ainsi, chaque année $n$, la cohorte de l'année antérieure $n$-I est en fait une cohorte tronquée et elle a été regroupée dans la cohorte $n-2$ : ainsi les mères nées en 1974 ont été regroupées dans la cohorte r973, les mères nées en 1973 dont les produits ont été contrôlés en 1974 ont été regroupées dans la cohorte I972; etc...

Les covariables considérées sont :

- le poids vif en fin de contrôle (voisin de $80 \mathrm{~kg}$ ) pour $\mathrm{X}_{1} \mathrm{X}_{2}$ et $\mathrm{X}_{3}$;

- le poids vif au moment des mesures pour $\mathrm{X}_{4}$ et $\mathrm{X}_{5}$.

b) Variables "descendants » $\left(\mathrm{Y}_{1} \grave{\mathrm{a}} \mathrm{Y}_{22}\right)$.

Un modèle linéaire additif a également été appliqué à ces données. Les effets principaux considérés sont :

- l'année de naissance (ou génération) du père qui va de $1965\left(\mathrm{G}_{0}\right)$ à 1974 $\left(G_{9}\right)$, sauf pour les variables $Y_{15}$ à $Y_{20}$ étudiées sur les 7 dernières générations seulement et les variables $\mathrm{Y}_{21}$ et $\mathrm{Y}_{22}$ étudiées sur les 6 dernières générations;

- la cohorte maternelle, définie comme précédemment, sauf pour les variables $\mathrm{Y}_{15}$ à $\mathrm{Y}_{20}$ étudiées sur I962-I973 et $\mathrm{Y}_{21}$ et $\mathrm{Y}_{22}$ sur I964-I973;

- le sexe avec 2 niveaux, femelle et mâle castré;

- un facteur milieu qui est :

pour les variables $\mathrm{Y}_{1}$ à $\mathrm{Y}_{14}$ une combinaison de l'année de contrôle et du bâtiment (3o niveaux),

pour les variables de qualité de viande $\mathrm{Y}_{15}$ à $\mathrm{Y}_{22}$, la date d'abattage (9r niveaux pour $\mathrm{Y}_{15}$ à $\mathrm{Y}_{20}$ et 65 niveaux pour $\mathrm{Y}_{21}$ et $\mathrm{Y}_{22}$ ).

c) Variables "loge» $\left(\mathrm{L}_{1}, \mathrm{~L}_{2}, \mathrm{~L}_{3}\right)$.

Les effets principaux du modèle utilisé sont pour ces variables :

- la génération du père (Io niveaux : $\mathrm{G}_{0}$ à $\mathrm{G}_{9}$ );

- le facteur milieu année-bâtiment (3o niveaux);

- le taux de masculinité de la loge, c'est-à-dire le pourcentage de mâles au début du contrôle (ro classes de pourcentage : 0-9, Io-I9, etc...). 
De plus les covariables suivantes ont été considérées :

- le poids moyen initial par porc (voisin de $30 \mathrm{~kg}$ );

- le poids moyen final (voisin de $80 \mathrm{~kg}$ );

- le nombre de morts ou d'éliminés.

L'analyse des variables $\mathrm{X}$ et $\mathrm{L}$ a été faite par le programme général de moindres carrés de HARVEY (I972) et celle des variables Y par un programme de moindres carrés du Département de Génétique animale.

\section{d) Estimation des tendances annuelles.}

Les évolutions observées pour toutes ces variables en fonction de la génération paternelle et de la cohorte maternelle ont été résumées par des régressions linéaires des estimées obtenues en fonction de l'année compte tenu des variances-covariances de ces estimées (voir Ollivier, I974, p. 48I). Ces régressions ont été doublés pour obtenir l'évolution moyenne des valeurs génétiques des reproducteurs correspondants, puisque les cohortes et générations successives sont comparées sur la valeur de leur descendance.

Tous les calculs ont été faits sur l'ordinateur IBM 370/I45 du Centre de Traitement de l'Information de Jouy-en-Josas.

\section{TABLEAU 3}

Analyse de la variance des variables "verrats " $\left(X_{1} \ldots X_{5}\right)$

(Analysis of variance of boar variables)

\begin{tabular}{|c|c|c|c|c|c|c|}
\hline \multirow{2}{*}{ Variable } & \multicolumn{4}{|c|}{$\begin{array}{c}\text { Carrés moyens : } \\
\text { Degrés de liberté entre parenthèses ( } \mathrm{I}) \\
\text { (Mean squares, degrees of freedom in parentheses) }\end{array}$} & \multirow{2}{*}{$\begin{array}{l}\text { Moyenne } \\
\text { estimée } \\
\text { (Least squares } \\
\text { mean) }\end{array}$} & \multirow{2}{*}{$\begin{array}{l}\text { Coefficient de } \\
\text { régression } \\
\text { (par } \mathrm{kg}) \\
\text { (Regression on } k g \\
\text { liveweight) }\end{array}$} \\
\hline & $\begin{array}{rr}\text { Année }(9) \\
(\text { Year) } & (8) \\
(2)\end{array}$ & $\begin{array}{r}\text { Cohorte (I } 4) \\
\text { Maternelle } \\
(\mathrm{I} 4) \\
(\text { Dam cohort) } \\
(7)\end{array}$ & $\mid \begin{array}{r}\text { Covariable } \\
(\mathrm{I}) \\
\text { Poids vif (I) } \\
(\text { Liveweight) } \\
(\mathrm{I})\end{array}$ & \begin{tabular}{|} 
Résiduels \\
$(825)$ \\
(Residual) \\
$(774)$ \\
$(142)$
\end{tabular} & & \\
\hline$X_{1}$ & $0,145\left(^{* *}\right)$ & $0,016 \mathrm{NS}$ & $0,449^{(* *)}$ & 0, OI I & $0,720 \pm 0,010$ & $0,010 \pm 0,002$ \\
\hline $\mathrm{X}_{2}$ & o,I $58 \mathrm{NS}$ & $0,181 \quad N S$ & $0,355 \mathrm{NS}$ & $0, \mathrm{II}_{2}$ & $3,22 \pm 0,06$ & $-0,02 \pm 0,01$ \\
\hline $\mathrm{X}_{3}$ & $89,9 \mathrm{I} \quad(* *)$ & $9,55 \mathrm{NS}$ & $240,45(* *)$ & 8,20 & $22,0 \quad \pm 0,4$ & $0,23 \pm 0,04$ \\
\hline$X_{4}$ & $23,76 \quad(* *)$ & $3,26 \mathrm{NS}$ & I $53,59\left(^{(* *)}\right.$ & 2,70 & $13,0 \pm 0,3$ & $0,25 \pm 0,03$ \\
\hline $\mathrm{X}_{5}$ & $30,02 \quad(* *)$ & $\left.7,86 \quad{ }^{*}\right)$ & I I $9,94\left({ }^{* *}\right)$ & 4,08 & $x 6,8 \pm 0,4$ & $0,19 \pm 0,03$ \\
\hline
\end{tabular}

$\mathrm{X}_{1}$ : Gain moyen quotidien (Average daily gain) $\mathrm{kg}$.

$\mathbf{X}_{2}$ : Indice de consommation (Feed conversion) $\mathrm{kg}$ aliment $/ \mathrm{kg}$ gain.

$\mathrm{X}_{3}$ : Épaisseur de lard vers $80 \mathrm{~kg}$ (Backfat thickness around $80 \mathrm{~kg}$ ) $\mathrm{mm}$.

$\mathrm{X}_{4}$ : Épaisseur de lard vers $40 \mathrm{~kg}$ (Backfat thickness around $40 \mathrm{~kg}$ ) $\mathrm{mm}$.

$\mathrm{X}_{5}$ : Épaisseur de lard vers $60 \mathrm{~kg}$ (Backfat thickness around $60 \mathrm{~kg}$ ) $\mathrm{mm}$.

(I) : lire de haut en bas les degrés de libertés respectifs de $\mathbf{X}_{1}$ et $\mathbf{X}_{3}$, de $\mathbf{X}_{4}$ et $\mathbf{X}_{5}$ et de $\mathbf{X}_{2}$ (From top to bottom, $X_{1}$ and $X_{3}, X_{4}$ and $X_{5}, X_{2}$ ).

NS : test $F$ non significatif; * : test $F$ significatif au seuil de 5 p. 100; ** : test $\mathrm{F}$ significatif au seuil de I p. roo 


\section{Résultats}

\section{I. - Analyse de variance}

Les résultats des analyses de variance sont donnés aux tableaux 3, 4, 5 et 6 respectivement pour les variables $\mathrm{X}, \mathrm{Y}$ et $\mathrm{L}$. Les différences observées entre mâles castrés et femelles sont toutes significatives à l'exception du poids de poitrine et du temps d'imbibition du muscle long vaste (tabl. 4 et 5). Cet effet significatif du sexe se retrouve au tableau 6 dans les effets du taux de masculinité à l'exception de la vitesse de croissance qui n'est pas significativement influencée par ce

\section{TABLEAU 4}

Analysis de la variance des variables "descendants " $\left(Y_{1} \ldots Y_{14}\right)$

(Analysis of variance of progeny variables)

\begin{tabular}{|c|c|c|c|c|c|c|}
\hline \multirow{3}{*}{ Variable } & \multicolumn{5}{|c|}{$\begin{array}{l}\text { Carrés moyens : Degrés de liberté entre parenthèses } \\
\text { (mean squares, degrees of freedom in parentheses) }\end{array}$} & \multirow{3}{*}{$\begin{array}{c}\text { Moyenne } \\
\text { estimée } \\
\text { (Least } \\
\text { squares } \\
\text { mean) }\end{array}$} \\
\hline & \multicolumn{4}{|c|}{ Effets principaux (Main effects) } & \multirow[b]{2}{*}{\begin{tabular}{|c|} 
Résiduels \\
$(2679)$ \\
$($ Residual $)$
\end{tabular}} & \\
\hline & $\begin{array}{l}\text { Sexe (I) } \\
(\operatorname{Sex})\end{array}$ & $\begin{array}{c}\text { Année- } \\
\text { Bâtiment } \\
(29) \\
\text { (Year- } \\
\text { building) }\end{array}$ & $\begin{array}{c}\text { Génération } \\
\text { paternelle } \\
(9) \\
(\text { Sive } \\
\text { generation) }\end{array}$ & $\begin{array}{c}\text { Cohorte } \\
\text { maternelle } \\
(\mathbf{1} 3) \\
(\text { Dam } \\
\text { cohort })\end{array}$ & & \\
\hline $\begin{array}{l}\text { G.M.Q. (A.D.G.). . . } \\
\text { Poids net (Carcass }\end{array}$ & I, I I $5(* *)$ & o, I I $9(* *)$ & $0,024(* *)$ & $0,025(* *)$ & 0,009 & 0,690 \\
\hline weight) & $45,64 \quad(* *)$ & $25,02 \quad(* *)$ & $8,12 \quad(* *)$ & $4,77 \quad(*)$ & $2,5 \mathrm{I}$ & 73,8 \\
\hline Tête (Head). . . & $7,28 \quad(* *)$ & $1,50 \quad(* *)$ & $0,58 \quad(* *)$ & $0,31 \quad(* *)$ & 0,14 & 5,06 \\
\hline Jambon (Ham) & $60,38 \quad(* *)$ & $0,86 \quad(* *)$ & $0,43 \quad(* *)$ & $0,17 \quad \mathrm{NS}$ & 0,16 & $8,2 \mathrm{I}$ \\
\hline Longe (Loin) & 292, I3 $\quad(* *)$ & $3,5^{8} \quad(* *)$ & $\mathrm{I}, 4 \mathrm{I} \quad(* *)$ & $0,7^{8} \quad(* *)$ & 0,34 & 10,52 \\
\hline Poitrine (Belly) & $0,25 \mathrm{NS}$ & $0,75 \quad(* *)$ & $0,30 \quad(* *)$ & $0,23 \quad(* *)$ & 0,09 & 3,96 \\
\hline Hachage (Shoulder) & $0,44 \quad(*)$ & $0,32 \quad(* *)$ & O,I $\left.7 \quad{ }^{*}\right)$ & o, I I NS & 0,09 & $5,5 \mathrm{I}$ \\
\hline Pieds (Feet). & $0,26 \quad(* *)$ & $0,12 \quad(*)$ & $0,02 \quad(* *)$ & o,oI NS & $0,0 \mathrm{I}$ & 0,96 \\
\hline Bardière (Back fat) & 646, I3 $\quad(* *)$ & $3,30 \quad(* *)$ & $3,21 \quad(* *)$ & $x, 53(* *)$ & 0,69 & 6,00 \\
\hline Panne (Leaf fat). & $12,62 \quad(* *)$ & $0,07 \quad(* *)$ & $0,20 \quad(* *)$ & $0,05 \mathrm{NS}$ & 0,04 & 0,94 \\
\hline $\begin{array}{l}\text { Longueur (Length). } \\
\text { Lard : }\end{array}$ & I92 $508(* *)$ & II $\left.3399^{* *}\right)$ & I $0_{4} \mathrm{I} N S$ & $2 \mathrm{I} 75(* *)$ & 868 & $97^{\circ}$ \\
\hline Rein (Rump fat). & I6 $804,8(* *)$ & 86,7 & 82,2 & I0o, 7 & 33,7 & 34,5 \\
\hline Dos (Back fat). & $9876,4\left(^{* *}\right)$ & 65,4 & $7 \mathrm{r}, \mathbf{I}$ & 46,9 & 20,2 & 29,0 \\
\hline Cou (Shoulder fat). & $8866, \mathrm{I}(* *)$ & $98,7 \quad(* *)$ & $99, \mathrm{I} \quad(* *)$ & $5 \mathrm{I}, 7 \mathrm{NS}$ & 32,9 & 52,5 \\
\hline
\end{tabular}

Les unités sont : le $\mathrm{kg}$ pour $\mathrm{Y}_{1}$ à $\mathrm{Y}_{10}\left(Y_{1}\right.$ to $Y_{10}$ in $\mathrm{kg}$ ).

le $\mathrm{mm}$ pour $\mathrm{Y}_{11}$ à $\mathrm{Y}_{14}\left(Y_{11}\right.$ to $Y_{14}$ in $\mathrm{mm}$ ). 


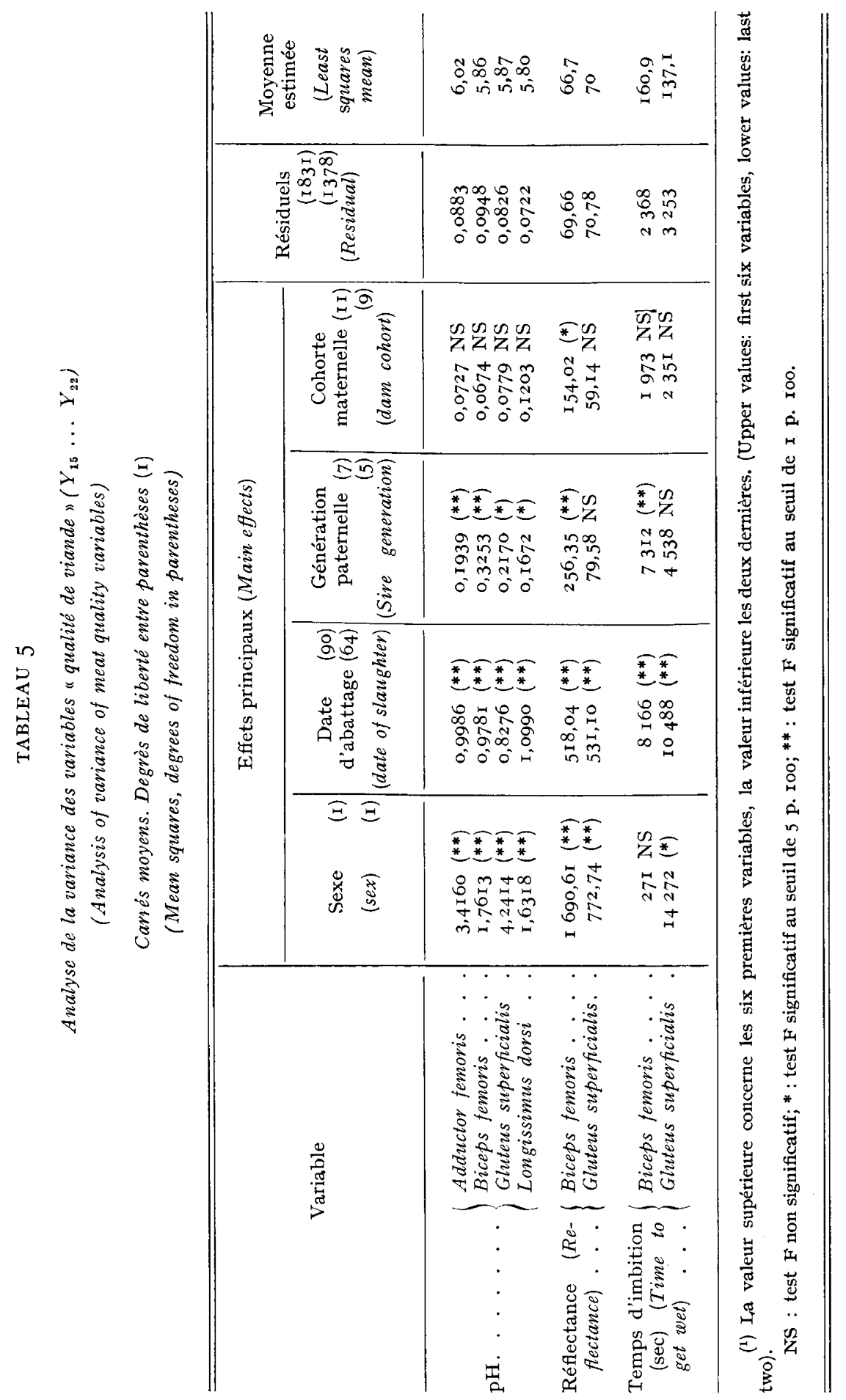


taux. Les effets de milieu des tableaux 4 à 6 (c'est-à-dire l'année, le bâtiment ou la date d'abattage selon les variables) sont tous hautement significatifs sauf pour le poids de hachage où le test $\mathrm{F}$ n'atteint que le seuil de $5 \mathrm{p}$. Ioo. Quant aux effets génétiques, leur ampleur, jugée par le niveau de signification des tests est différente selon qu'il s'agit des pères ou des mères. L'examen des tableaux 4 et 5 , où cette comparaison est possible, montre que les différences entre les générations paternelles successives sont significatives pour I9 variables sur 22, alors que les différences entre cohortes maternelles ne le sont que pour ro de ces variables. Les effets génétiques des tableaux 3 (cohorte maternelle) et 6 (génération paternelle) ne sont significatifs que pour l'épaisseur de lard à $60 \mathrm{~kg}$.

\section{TABLEAU 6}

Analyse de variance des variables "loge» $\left(L_{1}, L_{2}, L_{3}\right)$

(Analysis of variance of pen variables)

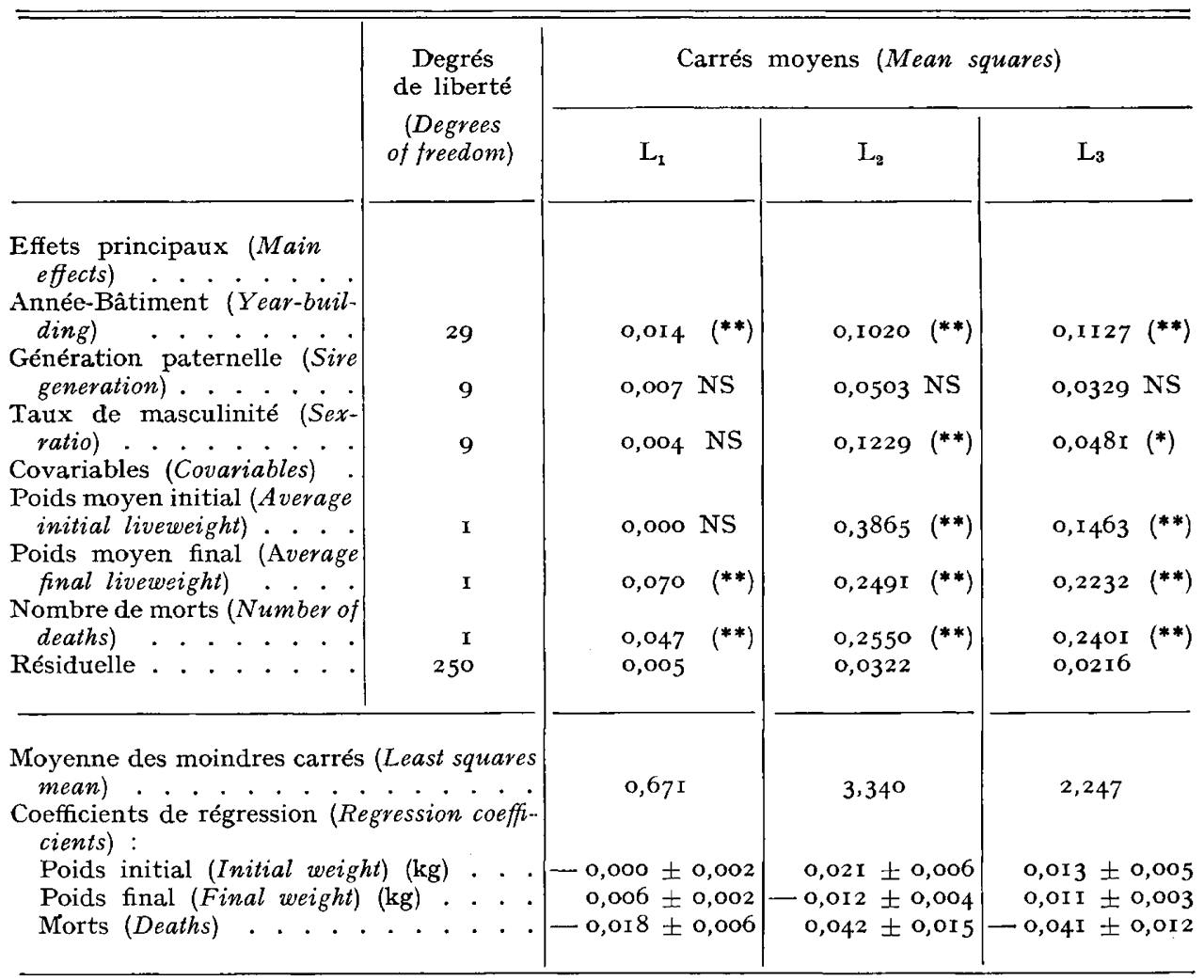

$\mathrm{I}_{11}$ : Gain moyen quotidien (Average daily gain) $\mathrm{kg}$ par porc.

$\mathrm{L}_{2}$ : Indice de consommation (Feed conversion) consommation totale de la loge/gain de poids total de la loge.

$\mathrm{L}_{3}$ : Consommation moyenne journalière (Daily feed intake) $\mathrm{kg}$ par porc. 
TABLEAU 7

Différences entre sexes

(Sex differences \pm standard error)

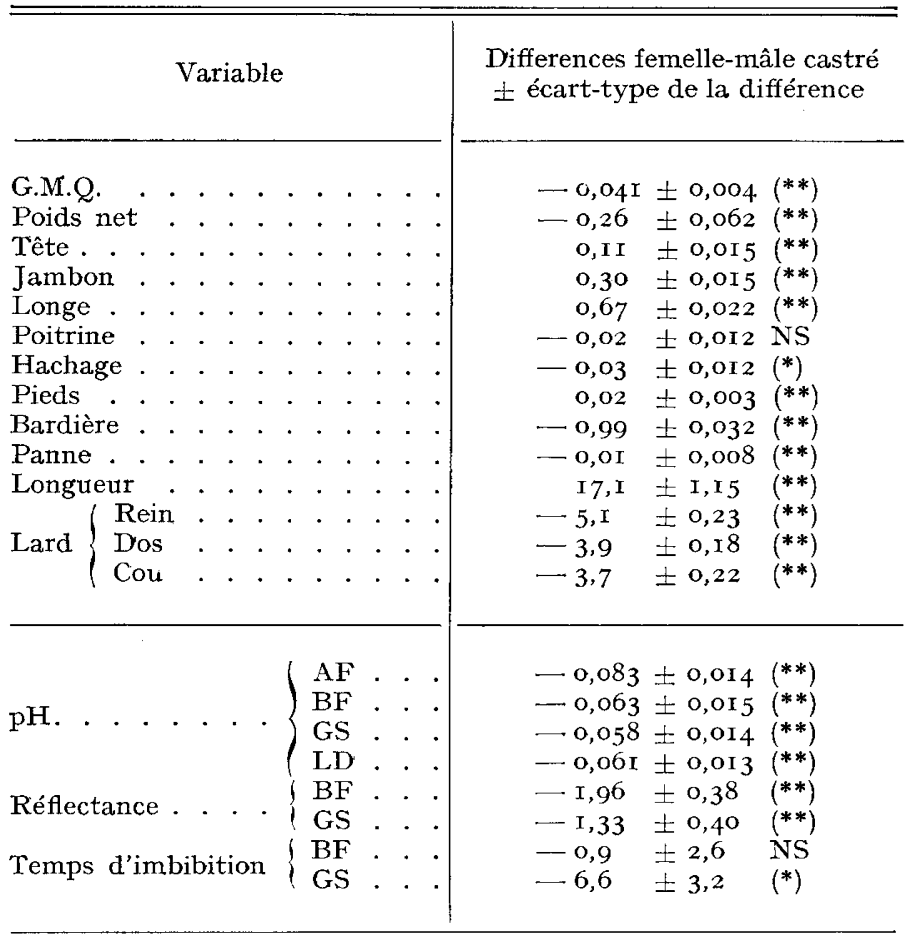

L,es variables sont celles des tableaux $4\left(\mathrm{Y}_{1} \ldots \mathrm{Y}_{14}\right)$ et $5\left(\mathrm{Y}_{15} \ldots \mathrm{Y}_{22}\right)$ avec les mêmes unités. (The variables are those of table 4 and 5 with the same units).

\section{2. - Estimations des effets}

Les effets " sexes » du tableau 7 montrent que les femelles ont par rapport aux mâles castrés, une croissance moins rapide, une carcasse plus riche en morceaux nobles, plus longue et moins grasse, et une viande plus acide, plus colorée (c'est-àdire à réflectance moins élevée) et plus humide.

Les valeurs comparées des générations paternelles et des cohortes maternelles sont représentées sous forme graphique pour les caractères de croissance et d'efficacité alimentaire (fig. I), pour les résultats de la découpe des carcasses (fig. 2), pour les mesures linéaires de carcasse (fig. 3) et enfin pour les mesures de qualité de viande (fig. 4). Pour ces dernières, les évolutions par muscle étant sensiblement parallèles, seules les moyennes, sur 4 ou 2 muscles, ont été représentées.

Les évolutions génétiques moyennes annuelles des pères et des mères sont données pour chaque caractère au tableau 8. Dans le calcul de toutes ces régressions 
les valeurs génétiques ont été exprimées en écart à la valeur de la première génération ou cohorte. Pour la plupart des variables de croissance et de carcasse les évolutions paternelles et maternelles concordent, les premières étant généralement plus rapides. Les évolutions de signe opposé concernent des variables à faible coefficient de régression pour au moins l'un des parents. C'est le cas de la tête, du

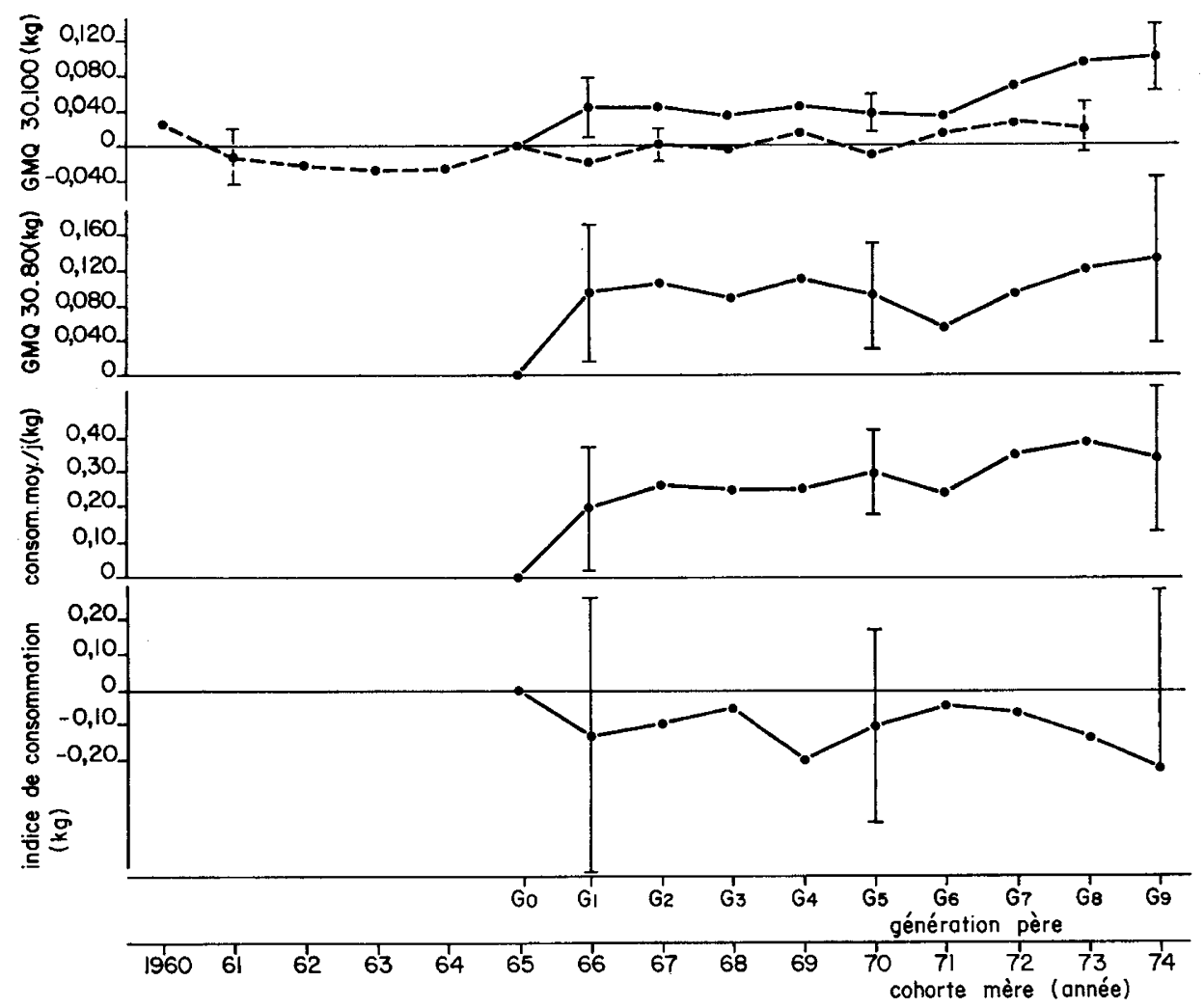

Fig: I. - Évolution génétique des caractères de croissance et d'efficacité alimentaire (Genetic trends for growth and feed efficiency).

génération paternelle (sive generation avec intervalle de confiance année de naissance de la mère (dam cohort) $\{$ à $95 \%$ (with 95 percent confi-...... génération paternelle (sire generation dence interval).

jambon, de la poitrine, du hachage, de la longueur, et des épaisseurs de lard au dos et au cou. Quant aux critères de qualité de la viande, leurs évolutions paternelles et maternelles sont toutes de signe opposé. Il faut cependant remarquer que, dans un sens comme dans l'autre, les régressions annuelles obtenues sont faibles relativement aux erreurs d'estimation, sauf pour la réflectance du biceps femoris du côté maternel. La couleur de ce muscle semble donc avoir évolué défavorablement sur la période I963-I973. 


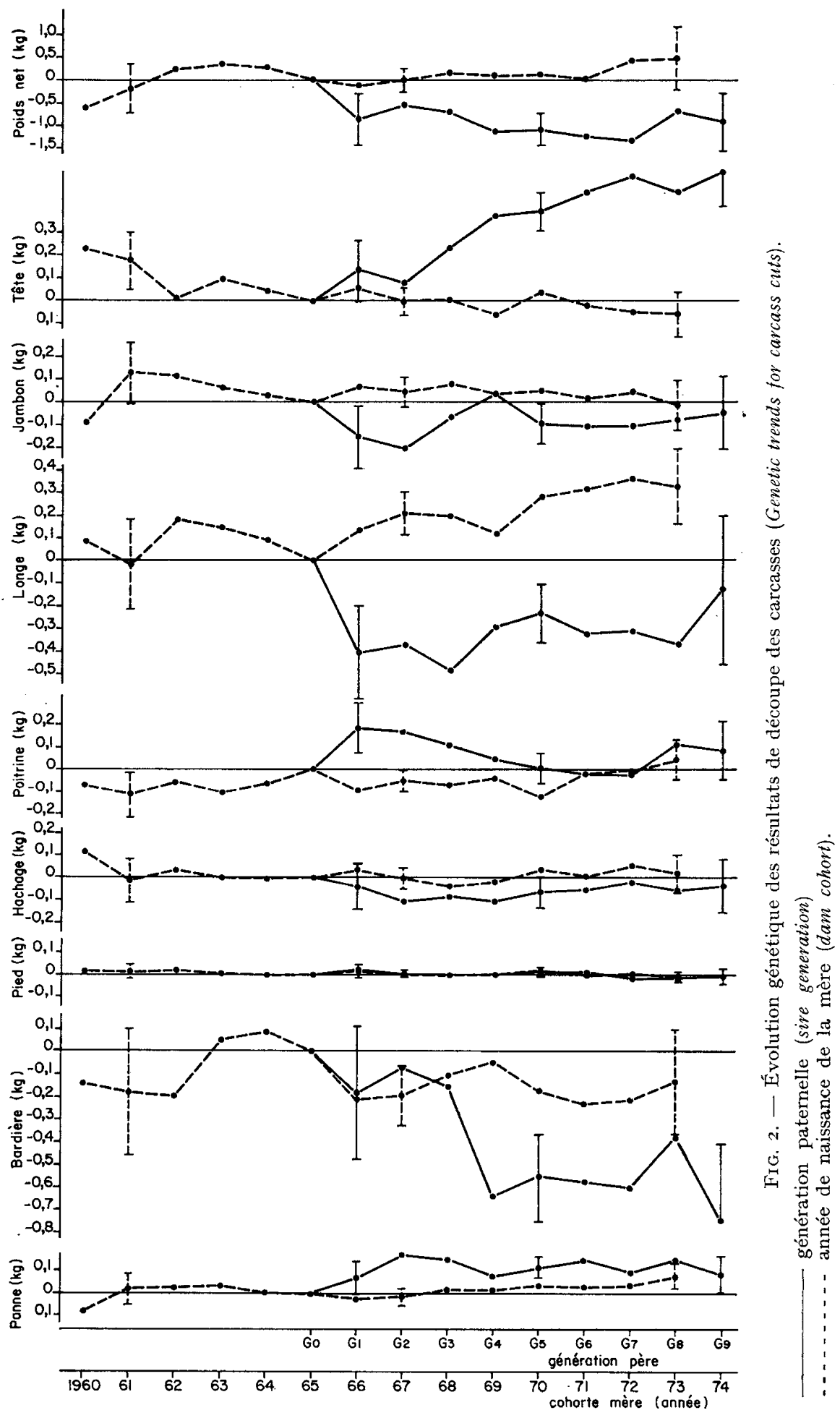




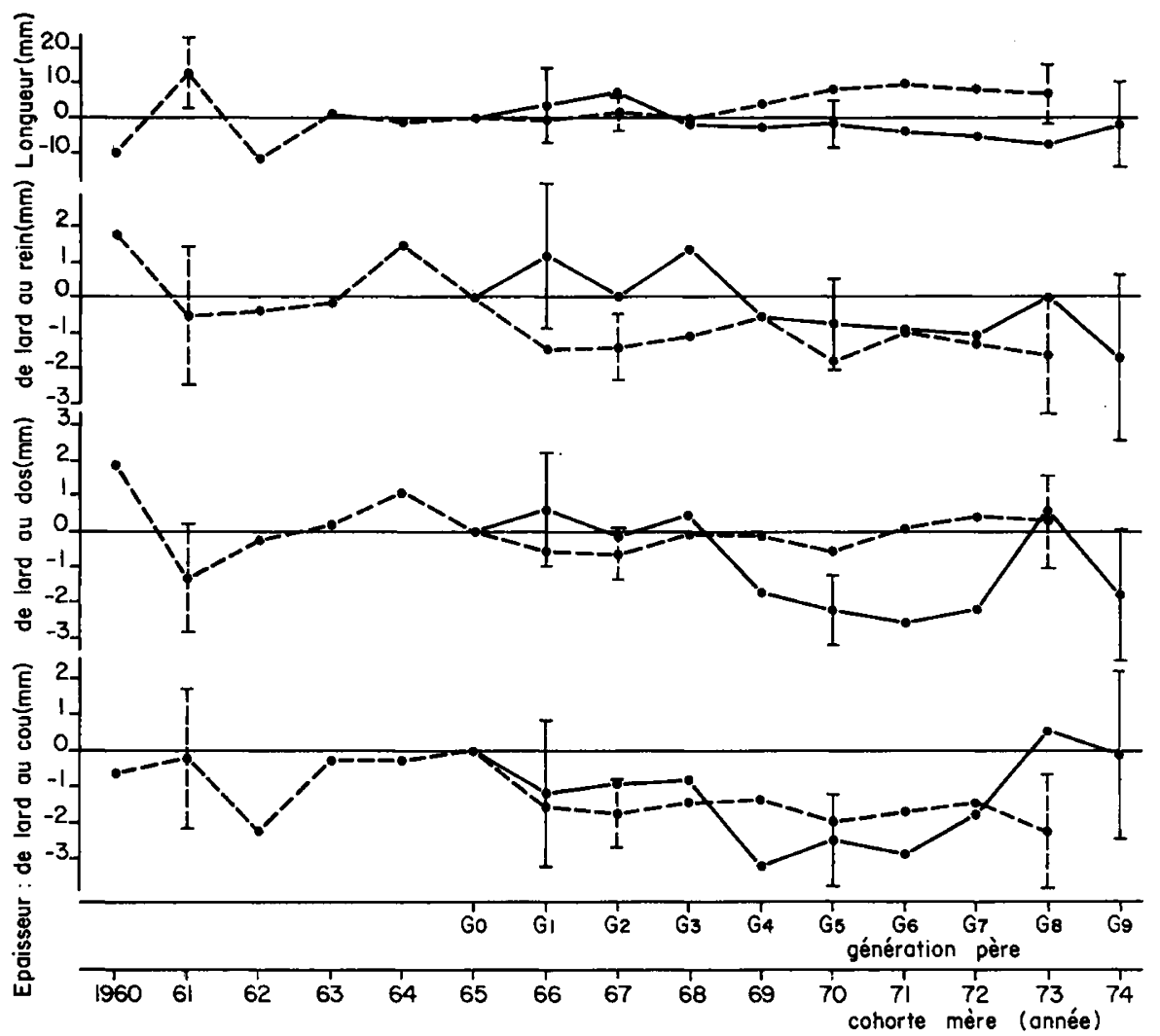

Frg. 3. - Évolution génétique des mesures linéaires de carcasse (Genetic trends for carcass linear measurements)

génération paternelle (sire generation)

année de naissance de la mère (dam cohort).

\section{Discussion et conclusions}

\section{I. - Modèles d'estimation}

La méthode d'estimation du progrès génétique utilisée dans cette expérience est analogue, dans son principe, à celle employée récemment dans le cas de verrats Large White soumis à l'épreuve de descendance en France de I953 à I966 (OlLIVIER, I974). Mais l'utilisation planifiée des verrats et l'insémination artificielle permettent ici, comme dans le dispositif expérimental similaire proposé par HICKMAN et FrEeman (I969), d'éliminer la plupart des risques inhérents à la méthode, à savoir les effets de la sélection des géniteurs et d'éventuels effets de milieu. Du côté paternel, les verrats éliminés après leur mise à la reproduction l'ont été pour cause d'accident ou d'inaptitude à l'insémination, et les effectifs de descendants par verrat ont été chaque année maintenus dans de faibles limites de variabilité. D'autre part, l'existence d'une éventuelle sélection a pu être vérifiée en 

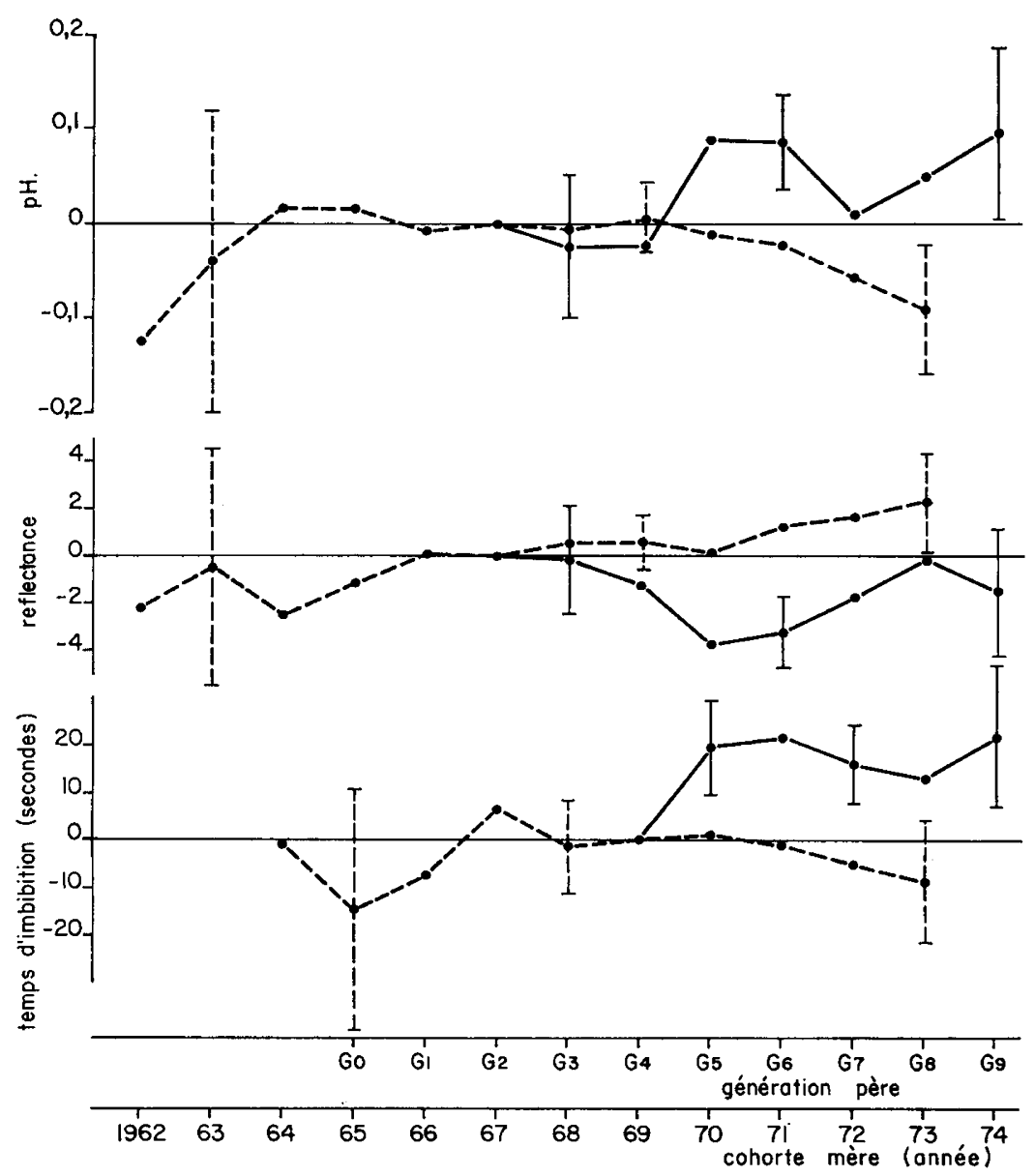

FIG. 4. - Évolution génétique des mesures de qualité de viande (Genetic trends for meat quality traits)

-_._._._. génération paternelle (sive generation)

........ année de naissance de la mère (dam cohort).

estimant les effets individuels correspondant aux 94 verrats de l'expérience. Les effets " génération " reconstitués à partir des moyennes des effets " père " sont très voisins de ceux obtenus en ignorant le facteur " père ". Par contre, la sélection du côté maternel n'est pas contrôlée dans ce schéma, et cela pourrait fausser les estimations d'amélioration génétique maternelle. Bien que les élevages utilisant l'insémination n'engraissent généralement pas les porcelets qu'ils produisent, et que de ce fait leurs possibilités de sélection soient limitées, un choix pourrait s'exercer après la mise à la reproduction des femelles principalement sur la vitesse de croissance, et son effet serait de sous-estimer les améliorations génétiques maternelles pour ce critère. Quant aux effets de milieu, les comparaisons faites, soit intra-bâtiment soit intra-date d'abattage, doivent minimiser leurincidence. Signalons cependant que l'alternance de loges de générations différentes dans 


\section{TABLEAU 8}

Évolutions génétiques annuelles paternelles et maternelles

(Annual sive and dam genetic trends)

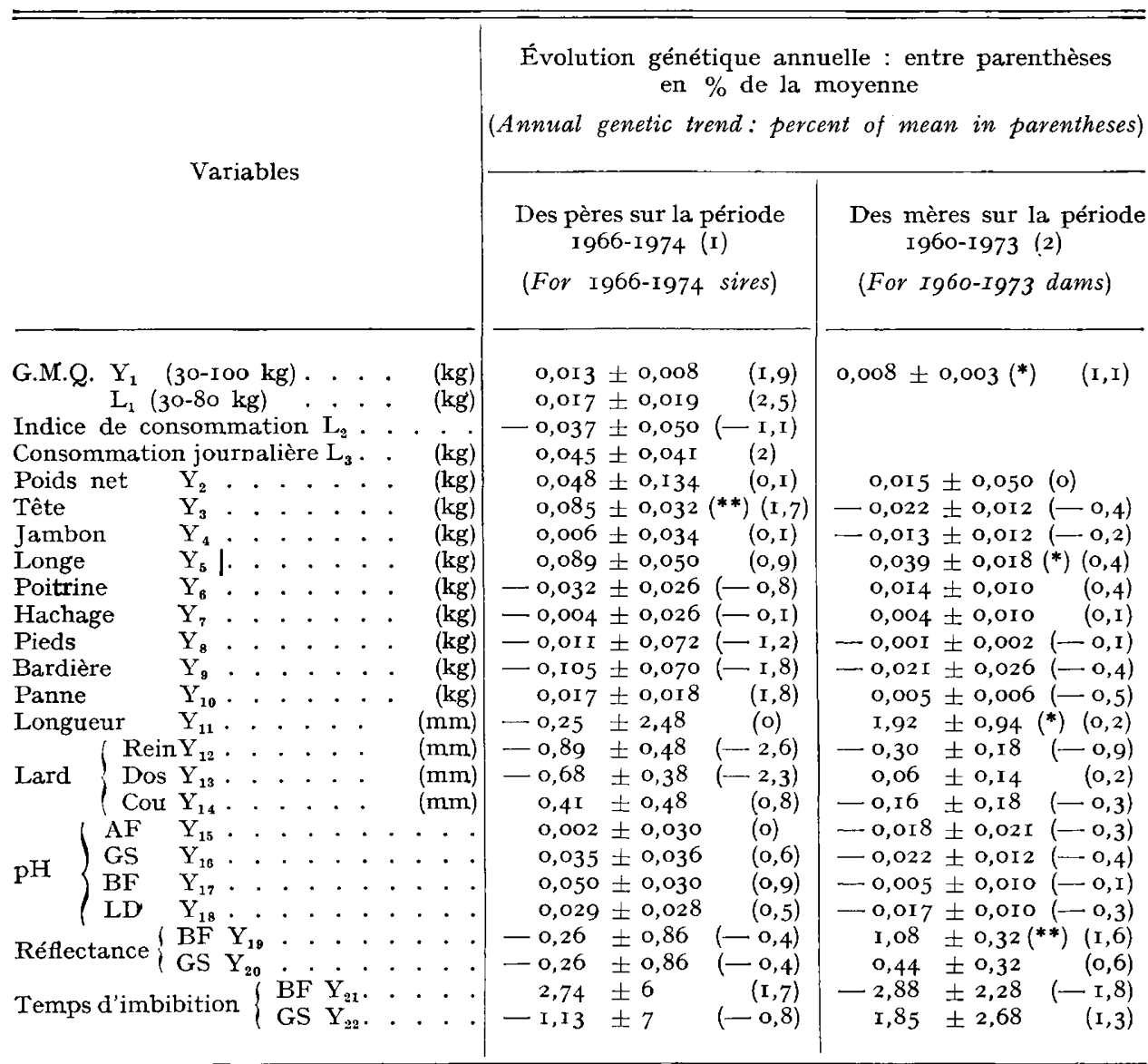

(1) La période considérée est réduite à I968-1974 pour $\mathrm{Y}_{15}$ à $\mathrm{Y}_{20}$ et I970-1974 pour $\mathrm{Y}_{21}$ et $\mathrm{Y}_{22}$. (The period considered is $1968-1974$ for $Y_{15}$ to $Y_{20}$ and $1970-1974$ for $Y_{21}$ and $Y_{22}$.

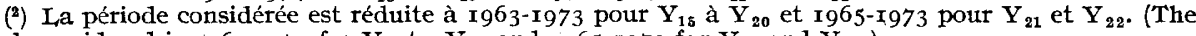
period considered is $1963-1973$ for $Y_{15}$ to $Y_{20}$ and $1965-1973$ for $Y_{21}$ and $Y_{22}$.)

chaque bâtiment n'a pas pu être réalisée lors de la première comparaison, faite en I967, entre $G_{0}$ et $G_{1}$. Cette comparaison n'a donc pas la rigueur des suivantes, mais cela n'entache pas la validité des régressions du tableau 8 , puisque l'effet $G_{0}$ a été éliminé pour le calcul de ces régressions. La comparaison des générations successives a pu également être faussée par le choix des mâles candidats à la sélection dans les portées de la génération la plus récente. De ce fait, malgré un effort d'équilibrage des sexes, le pourcentage de mâles dans les loges attribuées à cette génération était en moyenne inférieur à celui des loges de la génération antérieure. 
Cette distorsion a des effets importants pour au moins l'indice de consommation, comme le montre le tableau 6, mais ne doit pas fausser la comparaison des générations successives puisque ces effets sont éliminés dans l'analyse. Par contre, le choix des mâles à castrer était inévitablement basé en partie sur leur poids, ce qui a eu pour effet de créer une différence systématique de l'âge au début de contrôle (c'est-à-dire vers $30 \mathrm{~kg}$ ) entre les 2 générations comparées chaque année. Cet effet se retrouve dans l'analyse de cette variable (non incluse dans le tableau 4): l'âge à $30 \mathrm{~kg}$ subit une augmentation apparente sur les quatre premières générations paternelles de l'expérience, alors qu'il varie peu entre cohortes maternelles, cette dernière comparaison ne pouvant pas être affectée par le choix des mâles à castrer. Quoi qu'il en soit, la justesse des estimations doit en être peu affectée car les corrélations entre l'âge à $30 \mathrm{~kg}$ et les caractères mesurés ultérieurement sur l'animal sont très faibles (voir deuxième partie de l'article).

\section{2. - Effets non génétiques}

Les effets non génétiques mis en évidence aux tableaux 3 à 7 appellent quelques commentaires. Les variations importantes liées à la date d'abattage — qui va chaque année d'août à décembre - pour les critères de qualité de la viande (tabl. 5) traduisent l'influence prédominante des conditions climatiques, de transport et d'abattage sur ces critères, influence déjà signalée, entre autres, par JoNSSON (I965), Charpentier et al. (I97I), JAcQUeT et Ollivier (I97I), PfleiDERER (I973) et LUNDSTRÖM (I975). Les différences entre sexes du tableau 7 pour les I4 variables de croissance et de carcasse sont dans l'ensemble supérieures à celles précédemment trouvées (OLLIVIER, I974) dans les conditions d'une alimentation individuelle semi-ad libitum $(2,2 \mathrm{I} \mathrm{kg} /$ jour de 25 à Ioo $\mathrm{kg}$ contre $2,25 \mathrm{~kg} / \mathrm{jour}$ de 30 à $80 \mathrm{~kg}$ dans cette étude), ce qui semble confirmer que plus l'alimentation est intensive plus les différences entre sexes sont importantes (Jonsson, I965, I974). Une contradiction apparaît cependant entre les résultats du tableau 6 pour la vitesse de croissance de 30 à $80 \mathrm{~kg}$ et ceux du tableau 4 pour la vitesse de croissance de 30 à Ioo kg. Ces derniers montrent une différence entre sexes hautement significative, en faveur des mâles castrés, et conforme à la plupart des résultats de la littérature dans les conditions d'une alimentation à volonté. Par contre, quand on analyse la croissance moyenne des porcs d'une loge, celle-ci n'est pas significativement influencée par la proportion des mâles castrés dans la loge, et de plus, les estimées obtenues tendent au contraire à indiquer que la vitesse de croissance diminue quand cette proportion augmente : la tendance observée correspond en fait à une différence " apparente " de $43 \mathrm{~g} /$ jour en faveur des femelles. Cette inversion de la différence entre sexes (selon qu'on l'estime sur des individus de sexe différent placés dans la même loge ou à partir des variations de croissance moyenne des loges en fonction de la proportion de mâles castrés qui s'y trouvent) peut difficilement être attribuée à la différence dans les intervalles de poids considérés dans l'un et l'autre cas; elle pourrait résulter d'un effet dépressif sur la croissance du mélange d'animaux de sexe différent dans la même loge, dont on trouve par ailleurs des indications dans des résultats de BENKOv et al. (I973) et de BEKAERT et Casteels (I976).

Les différences de qualité technologique des viandes selon le sexe ont également été souvent étudiées, mais les résultats publiés sont dans l'ensemble moins concordants que pour les caractères d'engraissement et de carcasse. En effet, de nombreuses études n'ont pas mis en évidence d'effet significatif du sexe (voir 
WENiger et al., I967; SchMid, I968; BEKAERT et al., I968).Les différences significatives trouvées ici confirment cependant celles de OLLIVIER et MESLE (I963) pour l'acidité, de Charpentier et al. (I97I) et de Sellier (I975, I976, I977) pour l'acidité et la rétention d'eau. Ces différences vont toutes dans le sens d'une meilleure qualité technologique des viandes de mâles castrés. Les résultats concernant la couleur vont à l'encontre de cette tendance, puisque la viande des femelles est significativement plus colorée que celles des mâles castrés, ce qui confirme les résultats de Pease et Smith (I965), Kolaczy et Kotik (I966), Charpentier et al. (I97I) mais contredit ceux de Sellier (I975 et I977) et de Siers (I975). Des différences peu marquées entre sexes dans les notations de couleur ont été trouvées par Jonsson (I963, I965) de même que par JACQUET et Ollivier (I97I) pour des mesures de réflectance sur broyat de muscle BF frais et sur tranche de jambon cuit. Les causes possibles de ces discordances sont nombreuses (conditions d'abattage, muscle étudié, race, conditions d'élevage, méthode d'appréciation qui va selon les auteurs de la simple appréciation subjective au dosage des pigments contenus dans le muscle, la méthode objective la plus courante étant la réflectométrie) et il est difficile de se prononcer. Signalons aussi qu'une interaction sexe $\times$ concentration en énergie du régime a été récemment mise en évidence par GouTEFONGEA et al. (I977), les jambons des femelles ayant une qualité technologique supérieure à celle des mâles avec une alimentation à faible concentration en énergie.

\section{3. - Évolutions génétiques}

Les évolutions génétiques indiquées dans les figures I à 4 et au tableau 8 peuvent paraître relativement imprécises. Cependant les écarts-types des coefficients de régression, pour les variables $\mathrm{Y}_{\mathbf{1}}$ à $\mathrm{Y}_{\mathbf{1 4}}$, sont de l'ordre de 8 et $3 \mathrm{p}$. Ioo de l'écart-type résiduel respectivement pour les pères et pour les mères, chiffres à comparer aux Io p. Ioo en épreuve de descendance classique (Ollivier, I974) avec un effectif d'animaux supérieur de près de 50 p. Ioo à celui analysé ici. Le gain de précision que permet une application rigoureuse d'un schéma d'accouplements répétés est donc loin d'être négligeable. L'imprécision des estimations obtenues à partir des moyennes de loges $\left(\mathrm{L}_{1}, \mathrm{~L}_{2}, \mathrm{~L}_{3}\right)$ est soulignée par la comparaison, au tableau 8 , des erreurs sur $\mathrm{Y}_{1}$ et sur $\mathrm{L}_{1}$.

Deux raisons principales peuvent expliquer les différences (en vitesse ou en signe) des évolutions génétiques paternelles et maternelles. La première est le décalage des générations, qui sera discuté en détail dans la deuxième partie de cet article : en gros, les pères ont au moins un an et demi d'avance sur les mères contemporaines. La deuxième raison est la longueur de la période considérée, qui va de I960 à I 973 pour les mères et de I966 à I974 pour les pères. Le phénomène qui apparaît le plus clairement de ce fait est l'accélération de l'évolution génétique à partir de I966, début de l'expérience. Cela est particulièrement net pour la vitesse de croissance dont l'augmentation annuelle est presque doublée selon que l'on considère I960-I973 ou I965-I974. C'est encore plus net pour la composition corporelle. Le pourcentage de muscle dans une carcasse (sans tête) de poids $Y_{2}$ peut être estimé, à partir des poids d'un jambon $\left(\mathrm{Y}_{4}\right)$, d'une longe $\left(\mathrm{Y}_{5}\right)$ et d'une bardière $\left(\mathrm{Y}_{9}\right)$, par l'expression suivante, dérivée de celle donnée par HAMELIN (I975) pour la carcasse avec tête :

$$
\text { Muscle }(\%)=\mathrm{I} 8,82+\left(\mathrm{I} 43 \mathrm{Y}_{\mathbf{4}}+\mathrm{I} 66 \mathrm{Y}_{\mathbf{9}}-\mathrm{I} 53 \mathrm{Y}_{\mathbf{5}}\right) / \mathrm{Y}_{\mathbf{2}}
$$

où les variables sont exprimées en $\mathrm{kg}$. 
Le gain génétique annuel en pourcentage de muscle, autour de la moyenne qui est 46 p. Ioo, est la différentielle de (I) obtenue en reportant les estimations du tableau 8 comme différentielles de $\mathrm{Y}_{2}, \mathrm{Y}_{4}, \mathrm{Y}_{5}$ et $\mathrm{Y}_{\mathbf{9}}$. Ce gain est de 0,43 p. Ioo pour la période I966-I974 (pères) contre o,II p. Ioo pour la période I960-I973 (mères) : la vitesse d'évolution est donc environ multipliée par quatre pour la composition corporelle. Il faut cependant souligner que les évolutions moyennes annuelles du tableau 8 , surtout pour les valeurs génétiques paternelles, sont largement tributaires de la valeur atteinte par la dernière génération et donc affectées d'une assez grande imprécision. Il semble malgré tout que la tendance observée d'une accélération du progrès génétique depuis I966 ne puisse être mise en doute.

Ces résultats montrent que le mode de sélection employé dans cette expérience a permis d'accroître simultanément la vitesse de croissance, l'efficacité alimentaire et la production de tissu maigre. Pour synthétiser ces améliorations dans un critère global comme le rapport aliment consommé/tissu maigre produit, qui est l'indice de transformation en tissu maigre proposé par FowLER et al. (I976), il est nécessaire d'étendre à l'intervalle $30-100 \mathrm{~kg}$ les résultats sur l'indice de consommation $\left(\mathrm{L}_{2}\right)$ pour l'intervalle $30-80 \mathrm{~kg}$. En supposant que l'indice s'en trouve augmenté de 0,2 points (résultats non publiés), il a fallu en moyenne $(3,34+$ $0,2) /(0,738 \times 0,46)=I 0,42 \mathrm{~kg}$ d'aliment pour produire un kilogramme de maigre, et l'amélioration génétique annuelle (du côté paternel) a été de - o,2I kg d'aliment par kilogramme de maigre, soit environ 2 p. roo. Un autre critère proposé par les auteurs précédents est la vitesse de croissance du tissu maigre, qui a été en moyenne dans notre expérience $0,690 \times 0,738 \times 0,46=0,234 \mathrm{~kg} / \mathrm{jour}$ et s'est accrue annuellement (du côté paternel) de près de $7 \mathrm{~g} / \mathrm{jour}$.

La comparaison faite par SELLIER (I977) entre la dernière génération étudiée dans cette expérience $\left(\mathrm{G}_{9}\right)$ et le Large White national contemporain (élevages de sélection) complète les informations précédentes en situant les deux populations l'une par rapport à l'autre. Elles sont proches en efficacité de production du tissu maigre, puisque l'avantage du Large White avec abattage à $90 \mathrm{~kg}$, qui est de $0, \mathrm{I} 8 \mathrm{~kg}$ d'aliment par $\mathrm{kg}$ de tissu maigre produit, est compensé par un avantage similaire de la $\mathrm{G}_{9}$, de $0,24 \mathrm{~kg}$, quand l'abattage se fait à IIo kg. Par contre, la $\mathrm{G}_{9}$ est nettement supérieure au Large White en vitesse de croissance du tissu maigre, de 22 et $28 \mathrm{~g} /$ jour respectivement aux poids d'abattage de 90 et IIo $\mathrm{kg}$. Une supériorité de la $\mathrm{G}_{9}$ est également à noter pour l'ensemble des mesures de qualité de viande.

\section{4. - Comparaison avec d'autres expériences}

La seule expérience similaire à celle-ci qui nous soit connue est l'expérience norvégienn'e dont les résultats sur 5-6 générations ont été rapportés par VANGEN (I974), BAKKE (I975) et BAKKe et STANDAL (I975). L'expérience de l'ABRO à Edimbourg, sur une lignée synthétique ouverte pendant onze ans (WEBB et KING, I976) mérite aussi de retenir l'attention, en dépit des particularités du schéma expérimental qui rendent les comparaisons difficiles. Dans l'un et l'autre cas, la sélection était basée sur un indice, qui a cependant varié dans l'expérience de l'ABRO, et les réponses observées sont du même ordre de grandeur que celles rapportées ici (tabl. 9). L'ensemble de ces résultats ne confirme pas la crainte parfois exprimée (notamment par LISTER, I976) que la sélection contre l'adiposité aboutisse à réduire en fait l'efficacité alimentaire, à l'exemple du porc de Piétrain. Cette crainte n'est d'ailleurs pas non plus justifiée par les résultats obtenus quant à 
TABLEAU 9

Réponses par génération obtenues dans 3 expériences de sélection sur indice chez le Porc (Responses per generation in 3 experiments of index selection on swine)

\begin{tabular}{|c|c|c|c|}
\hline & $\begin{array}{c}\text { Expérience } \\
\text { norvégienne }\left({ }^{1}\right)\end{array}$ & $\begin{array}{l}\text { Expérience } \\
\text { de l'A.B.R.O. }\left(^{2}\right)\end{array}$ & $\begin{array}{c}\text { Présente } \\
\text { expérience }\left({ }^{3}\right)\end{array}$ \\
\hline Schéma expérimental (Experimental design) & $\begin{array}{l}\text { Lignée témoin } \\
\text { Lignée haute } \\
\text { Lignée basse }\end{array}$ & $\begin{array}{l}\text { Lignée synthéti- } \\
\text { que ouverte } \\
\text { sans témoin }\end{array}$ & $\begin{array}{l}\text { Accouplements } \\
\text { répétés }\end{array}$ \\
\hline Critères de sélection (Selection criteria). . & $\begin{array}{l}\text { Croissance et gras } \\
\text { dorsal }\end{array}$ & $\begin{array}{l}\text { I96o-1965 : lon- } \\
\text { gueur et épaiss } \\
\text { seur de lard } \\
\text { I966- I97 I : } \\
\text { croissance in- } \\
\text { dice de con- } \\
\text { sommation et } \\
\text { gras dorsal }\end{array}$ & Voir tableau $n^{\circ} \mathrm{I}$ \\
\hline Mode d'alimentation (Feeding regime). . & semi-ad libitum & ad libitum & ad libitum \\
\hline $\begin{array}{l}\text { Réponse moyenne par génération : entre } \\
\text { parenthèses en p. Ioo de la moyenne } \\
\text { (Response per generation: percent of } \\
\text { mean in parentheses) } \\
\text { - Gain moyen quotidien (g) (Average } \\
\text { daily gain). . . . . . . . . . . } \\
\text { - Indice de consommation (kg aliment } \\
\text { /kg gain) (Feed conversion) } \cdot \dot{\text {. }} \text {. } \\
\text { - Gras dorsal (mm) (Backfat thickness) }\end{array}$ & $\begin{array}{cc}5 & (0,8) \\
-0,027 & (0,9) \\
-0,9 & (3,1)\end{array}$ & $\begin{array}{cc}\text { I6 } & (2, I) \\
& \\
-0,046 & (1,4) \\
-0,6 & (2,5)\end{array}$ & $\begin{array}{cc}14 & (2,1) \\
-0,037 & (\mathrm{I}, \mathrm{I}) \\
-0,5 & (\mathrm{I}, 7)\end{array}$ \\
\hline
\end{tabular}

(1) VANGen (1974) pour le gain moyen quotidien et l'indice de consommation (5 générations). BAKKE et STANDAL (I975) pour le gras dorsal (6 générations).

$\left({ }^{2}\right)$ WebB et KING $(\mathbf{1 9 7 6})$ : moyennes par sexe et période pondérées par l'inverse de leur variance (période I960-I97I).

$\left({ }^{3}\right)$ Moyennes pondérées par l'inverse de leur variance pour les deux estimées de gain moyen quotidien et les trois estimées d'épaisseur de lard (période $1966-$ I974).

la vitesse de croissance dans trois expériences américaines de sélection sur l'épaisseur de lard (citées par VANGEN, I974).

Les évolutions en valeur relative du tableau 9 sont également intéressantes à considérer. Les deux expériences avec alimentation à volonté aboutissent à une amélioration de la vitesse de croissance nettement supérieure en valeur relative à celle observée pour l'efficacité alimentaire, ce qui correspond à une augmentation de l'appétit (que montre le tableau 8) qui n'apparaît pas dans l'expérience avec alimentation semi-ad libitum, celle-ci révélant en contrepartie une amélioration relativement plus importante de la production de tissu maigre. Ces évolutions ne contredisent pas celles prévues en théorie par FowLER et coll. (I976) 
en fonction de l'objectif et du milieu de sélection. Ils prévoient qu'une sélection sur la vitesse de croissance du tissu maigre avec une alimentation non restreinte (troisième cas p. 380) entraîne sur les premières générations peu de modifications de l'appétit. L'évolution de la consommation journalière d'aliment dans notre expérience entre $G_{1}$ et $G_{6}$ (fig. I) comme dans l'expérience norvégienne paraît conforme à ces prédictions. De même l'accroissement de consommation que nous observons à partir de $G_{7}$ pourrait correspondre à l'évolution prévue à plus long terme par les auteurs précédents.

\section{5. - Réponses indirectes}

Les effets d'une sélection du type précédent sur les qualités de la viande font l'objet de nombreuses spéculations. Une discussion détaillée du " dilemne qualité quantité " de la viande a été faite récemment par FredeEN (r975), qui a montré que les réponses à ce dilemne varient selon qu'on considère (I) l'évolution des populations porcines dans le temps (2) les différences entre races et (3) les variations et covariations génétiques à l'intérieur des races. L'expérience décrite ici contribue à fournir une réponse par la voie (I), contribution malheureusement limitée par le fait que la qualité de la viande n'a pas été mesurée dès le début de l'expérience. Les faibles vitesses d'évolution observées et le sens différent de l'évolution selon qu'on compare les 7 générations paternelles ou les Ir cohortes maternelles inclinent à penser que l'amélioration génétique dans la quantité de viande des carcasses n'a pas entraîné, au cours de la période considérée dans l'expérience, une dégradation de sa qualité, à l'exception peut-être de la couleur du long vaste. L'expérience norvégienne déjà citée permet à leurs auteurs d'exclure tout effet négatif sur la couleur du long dorsal du type de sélection pratiqué.

Il est intéressant de remarquer que le caractère dont l'évolution génétique paternelle est la plus nette (tabl. 8) est le poids de la tête qui s'est régulièrement accru au cours de l'expérience, une tendance qui avait aussi été relevée, mais avec moins de netteté, chez le Large White français de 1953 à Ig66 (Olurvier, I974). Il s'agit d'une fraction corporelle qui est rarement retenue parmi les critères de carcasse. Son évolution est à rapprocher de l'observation de Doornenbal (I975) selon lequel le tissu osseux n'atteint pas sa vitesse de croissance maximum avant $90 \mathrm{~kg}$ de poids vif chez le porc moderne, alors que ce maximum était atteint vers $20 \mathrm{~kg}$ dans les années 30 d'après les courbes de Hammond. Ainsi, le stade de maturité physiologique atteint à un même poids vif d'abattage de roo $\mathrm{kg}$ devient de moins en moins avancé, sous l'effet de la sélection contre l'adiposité, et le poids de tête peut être considéré comme un indicateur de cette maturité quand on sait qu'il représente 26 p. roo du poids vif à la naissance (PALsson, I955) et seulement $5 \mathrm{p}$. IOO au poids de Io $\mathrm{kg}$. On retrouve en fait dans cette expérience une évolution sur Io ans de l'importance relative de la tête analogue à celle mise en évidence en 1932 par Hammond (cité par Hammond $J r$ et al., r97 I) sur le porc PolandChina aux États-Unis entre I895 et I923, sous l'effet de la réduction de la demande de gras. Bien que dans notre expérience l'accroissement du poids de la tête n'ait pas eu de répercussion défavorable sur le rendement, contrairement à ce qui avait été observé en Large White de I952 à I966, cette éventualité n'est pas à exclure dans le long terme et tendrait à réduire l'efficacité de la production de tissu maigre. 


\title{
Remerciements
}

L'auteur remercie MM. F. du Mesnil du Buisson, et M. Couror (Département de Physiologie animale de l'I.N.R.A.) dont le soutien constant a permis la réalisation de cette expérience, M. F. Bariteau, Directeur de la Station expérimentale d'Insémination Artificielle de Rouillé (Vienne), pour le soin apporté avec l'aide de J. Bussik̀res, à la mise en service des jeunes verrats sélectionnés, et M. J. C. JEZEQUEL qui a assuré la responsabilité technique de l'expérience au cours des trois premières années. Les Établissements Archarmbault, à Celle-sur-Belle (Deux-Sèvres), doivent également être remerciés pour l'aide apportée dans les découpes de carcasse. L'auteur remercie enfin pour leurs observations les deux lecteurs du manuscrit, N. Standal (Agricultural University of Norway) et R. RouvieR (I.N.R.A, Toulouse).

\section{Summary}

\author{
A ten-year experiment on individual selection of boars \\ used in artificial insemination \\ I. - Observed responses on growth, carcass and meat quality traits
}

This paper describes the design and gives the results of a selection experiment carried on within a swine artificial insemination center, belonging to INRA, and located at Rouillé (Vienne, France). Starting from Io "founder boars" $\left(G_{0}\right)$, put into service in november 1965,9 successive boar generations $\left(G_{1}\right.$ to $\left.G_{9}\right)$ have been selected, i.e. 84 boars in all, and 2732 progeny (castrated males and females) from those 94 sires have been tested from 1966 to 1975 . The young boars put to insemination each year (in november) were sons of those selected one year before and of sows picked up at random in the insemination zone. Thus, no boar from outside this zone has been introduced during the whole experiment. The selection of boar was based on an individual index, equal to o.or $\mathrm{ADG}-0.5 \mathrm{BF}, \mathrm{ADG}$ being average daily gain in $\mathrm{g}$ from 30 to $80 \mathrm{~kg}$ liveweight and $\mathrm{BF}$ being backfat thickness in mm at $80 \mathrm{~kg}$. Individual feed intake was measured, and feed conversion included in the index, only up to 1968 . This boar performance testing was accompanied by a simultaneous comparison of the progeny from boars selected the year before and that from the remaining ones among those selected two years before (a so-called repeat-mating scheme). These pigs were fattened, in groups of 10 , with free access to self-feeders, from 30 to Ioo $\mathrm{kg}$ liveweight, in order to measure selection responses for growth rate, feed efficiency (on a pen basis), carcass and meat quality traits.

The data have been analysed by least-squares and for most variables, they show highly significant non-genetic effects, such as sex, building (for growth and carcass traits) or slaughtering date (for meat quality). Genetic differences, as a rule, are more important and more significant between sire generations than between dam cohorts (groups of sows born in the same year). As the periods investigated are 1960 to 1973 and 1966 to 1974 for dams and sires respectively, the more rapid trend observed over the sire generations indicates a speeding off in genetic change since 1966, probably due to the selection practised on the boars, the selection practised on the females by the individual farmers being supposedly very weak. Annual genetic gains on sires have been estimated as $\mathrm{I}_{4} \mathrm{~g}$ per day in growth rate, - 0.04 point in feed efficiency and $-0.5 \mathrm{~mm}$ in carcass backfat thickness. Percentage lean in carcass has been estimated from the weight of 3 cuts (ham, loin and backfat) and genetic gains have been derived for lean tissue feed conversion (LTFC) and lean tissue growth rate (LTGR): the annual sire trend is - $0.2 \mathrm{~kg}$ feed per $\mathrm{kg}$ lean tissue in LTFC and $7 \mathrm{~g}$ per day in LTGR. As to lean tissue quality, estimated through $\mathrm{pH}_{24}$ on longissimus dorsi and 3 muscles in the ham, colour and water-holding capacity on 2 muscles in the ham, there is no indication of a decline over the periods studied except for colour of biceps femoris, whose evolution, on the dam side, is unfavourable. A particularly remarkable trend is shown for weight of head, which has regularly and significantly increased from the beginning of the experiment. This indicates a lesser physiological maturity, at Ioo $\mathrm{kg}$ liveweight, of the fast growing lean animal favoured by the type of selection applied here. Consequently, a decrease in carcass weight is to be expected, though our results do not indicate any such decrease, 
The last generation included here $\left(G_{9}\right)$ has been compared to a sample of contemporary French Large White from nucleus herds by Sellier (Journées de la Recherche Porcine en France, I977, p. 85 ): the 2 strains are similar in $\mathrm{LTFC}$, but the $\mathrm{G}_{8}$ is superior to Lavge White in LTGR, by about $25 \mathrm{~g}$ per day, as well as in meat quality.

\section{Références bibliographiques}

BAKKe H., I975. Serum levels of non-esterified fatty acids and glucose in lines of pigs selected for rate of gain and thickness of backfat. Acta. Agric. Scand., 25, I I3-I 6.

BaKke H., Standal N., r975. Intramuscular fat content and some carcass composition traits in lines of pigs selected for rate of gain and thickness of backfat. Acta agric. Scand., 25, 22 I-224.

Bekaert H., Casteels M., I976. L'engraissement en groupes séparés de castrats et de truies du Landrace Belge et de la race Piétrain, avec application de l'alimentation à satiété et du rationnement. Rev. Agric. (Bruxelles), 29, 961-979.

Bezaert H., Eeckhout W., Casteels M., i968. Relations entre la qualité de la carcasse et la qualité de la viande chez les porcs. Rev. Agric. (Bruxelles), 21, I 473-I 49I.

Benkov M., Vasilev T., Stefanovas S., I973. Pig fattening as affected by their segregation by sex (en bulgare), Zhivotnovyd. Nauki, 10 (I), 89-92.

Charpentier J., Monin G., Ollivier L., i97I. Correlations between carcass characteristics and meat quality in Large White pigs. In: Proceedings of the 2 nd intern. Symposium on Condition and Meat Quality of Pigs, 255-26o, Pudoc, Wageningen.

Doornenbal H., 1975. Growth, development and chemical composition of the pig. IIr. - Bone, ash and moisture. Growth, 39, 427-434.

Dumont B. L., I957. Nouvelles méthodes pour l'estimation de la qualité des carcasses sur les porcs vivants. Réunion de la F.E.Z. sur l'épreuve de la descendance des porcs. Copenhague.

Fowler V. R., Bichard M., Pease A., i976. Objectives in pig breeding. Anim. Prod., 23, $365-387$.

Fredeen H. T., I958. Selection and swine improvement. Anim. Breed. Abstr., 26, 229-24I.

FREDEEN H. T., I975. Future aspects in breeding a modern meat-type pig. In: Fetskrift til Hjalmar Clausen, 49-7I, Det Kgl. Danske Landhusholdningsselskab, Copenhague.

Goodwin K., Dickerson G. E., Lamoreux W. F., I955. A technique for measuring genetic progress in poultry breeding experiments. Poult. Sci., 34, I 197.

Goutefongea R., JaCQuer B., Sellier P., I977. Influences génétiues et non génétiques sur l'aptitude du jambon à la transformation. $23^{\mathrm{e}}$ Congrès Eur. des chercheurs en Viande, Moscou, 4-9 sept. $1977,8 \mathrm{p}$.

Hamelin M., 1975. (Communication personnelle).

Hammond Jr. J., Mason I. L., Robinson T. J., I971. Hammond's Farm Animals. Edward Arnold, Londres.

Hanset R., VAN Snick G., 1972. Les paramètres génétiques des caractères d'engraissement et de carcasse chez le porc de Piétrain. Ann. Génét. Sél. anim., 4, 451-467.

HANSET R., VAN SNick G., 1973. Les paramètres génétiques des caractères d'engraissement et de carcasse chez le porc Landrace Belge. Ann. Génét. Sél. anim., 5, 369-379.

HARVEY W. R., I972. Least-squares and maximum-likelihood general purpose program. Ohio State University.

HAZEL L. N., KLINE E. A., I952. Mechanical measurement of fatness and carcass value on live hogs. J. Anim. Sci., 11, 3I3-3I8.

Hetzer H. O., Miller L. R., I973. Selection for high and low fatness in swine. Correlated responses of various carcass traits. J. Anim. Sci., I 289-I 301 .

Hickman C. G., Freeman A. E., I969. New approach to experimental designs for selection studies in dairy cattle and other species. J. Dairy Sci., 52, I 044-I o54.

Jacguet B., Ollivier L., r971. Résultats d'une expérience de croisement Piétrain $\times$ Large White. II. - Aptitude du jambon à la transformation en jambon de Paris. In: Journées de la Recherche Porcine en France, I97I, 23-33, Institut technique du Porc, Paris.

Jonsson P., r963. Danish pig progeny testing results. Z. Tievz. Zucht Biol., 78, 205-252.

Jonsson P., 1965. Analyse af egenskaber hos svin af Dansk Landrace med en historisk indledning. 350. beretning fra forsøgslaboratoriet, $490 \mathrm{pp}$. 
Jonsson P., 1974. Applied aspects of pig selection. In : I er Congrès mondial de Génétique appliquée à l'Élevage, vol. I, 837-847.

Kolaczy K S., Kotıк T., I 966 . A note on sex differences in muscle properties of pigs with special emphasis on colour. Anim. Prod., 8, I 53-154.

LisTeR D., 1976. Hormonal influences on the growth, metabolism and body composition of pigs. In: Lister D., Rhodes D. N., Fowler V. R., Fuller M. F., Meat Animals, Growth and Productivity, 355-373, Plenum Press, New-York.

Lundström K., I975. Genetic parameters estimated on data from the Swedish pig progeny testing stations with special emphasis on meat colour. Swedish J. agric. Res. 5, 209-22 I.

Oleivier L., I965. Accroissement en épaisseur du tissu gras dorsal chez le verrat en croissance. Ann. Zootech., 14, 39I-400.

Ollivier L., I970 a. L'épreuve de la descendance chez le porc Lavge White français de I953 à 1966. I. - Analyse de la variation. Ann. Génét. Sél. anim., 2, 31 I-324.

Ollivier L., i97o $b$. Premiers résultats d'une expérience de sélection réalisée dans un centre d'insémination arțificielle porcine. ${ }_{21}{ }^{\mathbf{e s}}$ Journées d'études de la Féd. eur. Zootech., Budapest, 24-28 Août 1970, 7 p.

Ollivier L., r97I. Premiers résultats d'une expérience de sélection réalisée dans un centre d'insémination artificielle porcine. In: Journées de la Recherche Porcine en France 1971, 35-40, Institut technique du Porc, Paris.

Ollivier L., 1974. L'épreuve de la descendance chez le Porc Lavge White français de 1953 à 1966. III. - Progrès génétique. Ann. Génét. Sél. anim., 6, 477-492.

Ollivier L., Mesle L., I963. Résultats d'un contrôle de descendance portant sur la qualité de la viande chez le porc. Ann. Zootech., 12, I73-179.

Palsson H., I955. Conformation and body composition. In: Progress in Physiology of Farm Animals, 430-542, HAMmond J. (ed.), Butterworth, Londres.

Pease A. H. R., Smirh C., 1965. A note on the heritability of muscle colour in pigs. Anim. Prod., 7, 273-274.

Pfleiderer U. E., 1973. Genetische Parameter der wichtigsten Mastleistungs - und Schlachtkörpermerkmale aus der Stationsprüfung von Schweinen der Deutschen Landrasse. I. Heritabilitätsschatzungen. Züchtungskunde, 45, 21 5-223.

Pfleiderer U. E., 1974. Genetische Parameter der wichtigsten Mastleistungs - und Schlachtkörpermerkmale aus der Stationsprüfung von Schweinen der Deutschen Landrasse. II. Genetische Korrelationen und möglicher Selektionserfolg. Züchtungskunde, 46, I59-167.

RAHNEFeld G., 197I. Mass selection for post-weaning growth in swine. II. - Response to selection. Can J. Anim. Sci., 51, 497-502.

Scнмid P., I968. Beobachtungen uber pH-Wert und Farbe von Schweinefleisch. Schweiz. landw. Monatsh., 46, 349-356.

Sellier P., I975. Valeur en croisement de verrats Landrace Français et Piétrain. In: Journées de la Recherche Porcine en France, 1975, 285-29I, Institut technique du Porc, Paris.

Sellier P., 1976. Valeur en croisement de verrats Landrace Belge et Piétrain. In: Joumées de la Recherche Porcine en France, 1976, 221-228, Institut technique du Porc, Paris.

Sellier P., I977. Valeur en croisement de verrats Lavge White et Piétrain; influence du poids d'abattage. In: Journées de la Recherche Porcine en France, I977, 85-89, Institut technique du Porc, Paris.

SiERS D. G., 1975. Live and carcass traits in individually fed Yorkshire boars, barrows and gilts. J. anim. Sci., 41, 522-526.

Smith C., King J. W. B., Gilbert N., 1962. Genetic parameters of British Large White bacon pigs. Anim. Prod., 4, I28-ז43.

VANGEN O., I974. Growth rate and feed conversion in lines of pigs selected for rate of gain and thickness of backfat. Acta. Agric. Scand., 24, 309-318.

WebB A. J., KING J. W. B., I976. Development of a synthetic pig sire line by selection with immigration. I. - Results of selection and heritability estimates. Anim. Prod., 22, 23I-244.

Weniger J. H., Steinhauf D., Glodek P., Weiss F. K., 1967. Einige Merkmale der Fleischbeschaffenheit beim Schwein, ihre Erblichkeit, ihre Beziehungen zu anderen Leistungseigenschaften und ihre Abhängigkeit von der Witterung. Zuchtungskunde, 39, 332-345. 